\title{
Saltzman's Model. Part I: Complete Characterization of Solution Properties
}

\author{
S. LAKSHMIVARAHAN \\ School of Computer Science, University of Oklahoma, Norman, Oklahoma \\ JOHN M. LEWIS \\ National Severe Storms Laboratory, Norman, Oklahoma, and Desert Research Institute, Reno, Nevada \\ JUNJUN HU \\ Cooperative Institute for Mesoscale Meteorological Studies, University of Oklahoma, Norman, Oklahoma
}

(Manuscript received 17 November 2017, in final form 15 November 2018)

\begin{abstract}
In Saltzman's seminal paper from 1962, the author developed a framework based on the spectral method for the analysis of the solution to the classical Rayleigh-Bénard convection problem using loworder models (LOMs), LOM $(n)$ with $n \leq 52$. By way of illustrating the power of these models, he singled out an LOM (7) and presented a very preliminary account of its numerical solution starting from one initial condition and for two values of the Rayleigh number, $\lambda=2$ and 5 . This paper provides a complete mathematical characterization of the solution of this LOM (7), herein called the Saltzman LOM (7) [S-LOM (7)]. Historically, Saltzman's examination of the numerical solution of this low-order model contained two salient characteristics: 1) the periodic solution (in the physical 3D space and time) that expand on Rayleigh's classical study and 2) a nonperiodic solution (in the temporal space dealing with the evolution of Fourier amplitude) that served Lorenz in his fundamental study of chaos in the early 1960s. Interestingly, the presence of this nonperiodic solution was left unmentioned in Saltzman's study in 1962 but explained in detail in Lorenz's scientific biography in 1993. Both of these fundamental aspects of Saltzman's study are fully explored in this paper and bring a sense of completeness to the work.
\end{abstract}

\section{Introduction}

Thermally induced convection in response to a fluid heated from below has found many applications in meteorology. Among the events that stem from this process are buoyant plumes of air adjoining the ground or sea surface in response to solar heating of that surface, sea breezes that are generated through differential solar heating of sea and adjoining land surfaces, and the formation of stratus cloud over the ocean in the presence of warm sea surface temperature. The classical Oberbeck and Boussinesq approximation (Saltzman 1962) has provided the mathematical basis for the analysis of convection arising from thermal instability for well over a century. In addition to providing a historical account of the developments, Chandrasekhar (1961) contains a

Corresponding author: S. Lakshmivarahan, varahan@ou.edu thorough analysis of the linear version of the thermally induced convection problem.

There are essentially two different routes to solve the system of nonlinear coupled partial differential equations (PDEs) arising from the above said approximation. The first is to numerically simulate convection using a suitable space-time grid. The second is to capture the spatial variations to any desired degree of accuracy using a finite number of Fourier modes and reduce the PDE to an initial value problem consisting of $n$ coupled nonlinear ordinary differential equations (ODEs). This resulting system of coupled nonlinear equations is called a low-order model (LOM) of order $n$ and is denoted by LOM (n). This latter approach is known as the Galerkin projection method or simply a spectral method (Canuto et al. 2007; Shen et al. 2011).

Early applications of both the space-time gridpoint method and the spectral method to the convection 
problem centered on linear dynamics and steady-state solutions. A nearly complete list of contributions to the problem up through the early 1970s is found in J. S. Turner's classic treatise on buoyancy in fluids (Turner 1973, chapter 7). Prior to Saltzman's (1962) contribution, a theoretical paper by Malkus and Veronis (1958) established the two-dimensional spectral framework that he followed.

Saltzman (1962) meticulously developed the structure of a family of LOM $(n)$ for $n \leq 52$. This family of models has been the basis for numerous subsequent studies. The well-known LOM (3), known as the Lorenz 1963 model (Lorenz 1963), is a member of this family of models as are the ones in Curry (1978).

By way of illustration, Saltzman (1962) concluded his paper with a preliminary and incomplete analysis of a seven-mode model, called the Saltzman LOM (7) [S-LOM (7)]. If Lorenz's biography (Lorenz 1993) is any guide, using this LOM (7), Saltzman seems to have demonstrated the existence of a nonperiodic solution to Lorenz at the time when he visited Saltzman in 1961.

Despite the importance of this chaotic aspect of Saltzman's LOM (7), it is surprising that he failed to mention it in his treatise. Yet we know from Lorenz's scientific biography (Lorenz 1993, p. 137) that Saltzman was aware of this important feature. Although Lorenz (1993) gives full credit to Saltzman for showing him the preliminary nonperiodic results that were critical to his seminal paper, we the readership of Lorenz (1963) were unaware of this important interaction of the fellow classmates at MIT in the 1950s.

Thus, there was a strong motivation to conduct a full-scale examination of the properties of S-LOM (7). Our analysis reveals that S-LOM (7) exhibits an inherent competition between the amplification of energy resulting from the nonlinear interaction and dissipation resulting from the friction terms. For initial conditions (ICs) close to the origin, the amplification component dominates, and the solution $\mathbf{X}(t)$ grows in time. Once $\mathbf{X}(t)$ grows to a sufficient level, the dissipation part takes control leading to asymptotic convergence in one of the four (two in each of the invariant subspaces $\mathrm{IS}_{1}$ and $\mathrm{IS}_{2}$ ) $1 \mathrm{D}$ equilibrium manifolds described below. It turns out that the S-LOM (7) can be decomposed into a union of two copies of Lorenz-like LOM (3) and one copy of a linear LOM (3), which are interconnected by a set of nine nonlinear coupling terms. It turns out that each of these two Lorenz-like subsystems and the linear LOM (3) define three invariant subspaces for S-LOM (7). The solution $\mathbf{X}(t) \in R^{7}$ of the S-LOM (7), depending only on the distribution of energy in the initial condition and the value of the parameter $\lambda$, finds itself in one of the three invariant subspaces.

The asymptotic behavior of $\mathbf{X}(t)$ in these invariant subspaces is essentially dictated by the rotation symmetry of the S-LOM (7) projected onto these invariance subspaces. Two of the invariant subspaces, labeled $\mathrm{IS}_{1}$ and $\mathrm{IS}_{2}$, each admit two branches of $1 \mathrm{D}$ equilibrium manifolds parameterized by $\lambda$. For the third invariant subspace $\mathrm{IS}_{3}$, the origin is the only stable attractor. Finally, we bring out the multifaceted behavior of S-LOM (7) using a deterministic ensemble experiment by starting the solution from the 128 corners of a sevendimensional hypercube centered at the origin with sides of length $2 \alpha$ for $\alpha \in(0,1]$.

There is a close connection between the class of energy conserving LOM $(n)$ and the system of Volterra gyrostats and their generalization. Refer to Gluhovsky and Tong (1999), Lakshmivarahan and Wang (2008), and Tong (2009) for details.

In section 2, we identify the three invariant subspaces and the equilibria contained in them. The stability properties of these equilibria are developed in section 3. Section 4 contains an analysis of the global properties of the solution of S-LOM (7). Results of the deterministic ensemble and other experiments are contained in section 5. A concluding summary is given in section 6. Detailed analysis of the stability of the isolated equilibrium at the origin is contained in appendix A. Analysis of the boundedness of the solution is given in appendix B. Appendix C describes a simple encoding scheme to represent the 128 corners of the seven-dimensional hypercube used in the ensemble analysis given in section 5. Finally, in appendix D, we describe a linear invertible transformation, using which we prove the equivalence between the Lorenzlike LOM (3) in IS $_{1}$ and the Lorenz (1963) model with $\sigma=10$ and $\beta=8 / 3$.

\section{Analysis of Saltzman's model}

The equations of S-LOM (7) may be stated as follows. Let $\mathbf{X}=\left(X_{1}, X_{2}, \ldots, X_{7}\right)^{\mathrm{T}} \in R^{7}$ and $\mathbf{f}: R^{7} \times R \rightarrow R^{7}$, the S-LOM (7) is given by

$$
\dot{\mathbf{X}}=\mathbf{f}(\mathbf{X}, \lambda),
$$

where $\mathbf{f}=\left(f_{1}, f_{2}, \ldots, f_{7}\right)^{\mathrm{T}}$ is the vector field and $\lambda>0$ is the Rayleigh number. From pages 336 and 340 in section 7 in Saltzman (1962), $\lambda=R / R_{c}$ is a control parameter, and the components of this vector field (after relabeling: $A=X_{1}, B=X_{2}, C=X_{3}, D=X_{4}, E=X_{5}, F=X_{6}$, $\left.G=X_{7}\right)$ are given by 
TABLE 1 . Values of coefficients $C_{i j k}$.

\begin{tabular}{llll}
\hline \hline$C_{123}=23.521$ & $C_{213}=-22.030$ & $C_{312}=1.561$ & $C_{714}=27.916$ \\
$C_{140}=-1.500$ & $C_{250}=-1.589$ & $C_{360}=-0.185$ & $C_{725}=37.220$ \\
$C_{110}=-148.046$ & $C_{220}=-186.429$ & $C_{330}=-400.276$ & $C_{770}=-39.479$ \\
$C_{435}=-16.284$ & $C_{534}=16.284$ & $C_{615}=16.284$ & $C_{624}=16.284$ \\
$C_{426}=-16.284$ & $C_{516}=-16.284$ & $C_{630}=-486.877$ & \\
$C_{417}=-13.958$ & $C_{527}=-18.610$ & $C_{660}=-40.028$ & \\
$C_{440}=-14.805$ & $C_{520}=-1947.508$ & & \\
$C_{410}=-1460.631$ & $C_{550}=-18.643$ & & \\
\hline
\end{tabular}

$$
\begin{aligned}
& f_{1}(\mathbf{X}, \lambda)=C_{123} X_{2} X_{3}+C_{140} X_{4}+C_{110} X_{1}, \\
& f_{2}(\mathbf{X}, \lambda)=C_{213} X_{1} X_{3}+C_{250} X_{5}+C_{220} X_{2}, \\
& f_{3}(\mathbf{X}, \lambda)=C_{312} X_{1} X_{2}+C_{360} X_{6}+C_{330} X_{3}, \\
& f_{4}(\mathbf{X}, \lambda)=C_{435} X_{3} X_{5}+C_{426} X_{2} X_{6}+C_{417} X_{1} X_{7}+C_{410} \lambda X_{1}+C_{440} X_{4}, \\
& f_{5}(\mathbf{X}, \lambda)=C_{534} X_{3} X_{4}+C_{516} X_{1} X_{6}+C_{527} X_{2} X_{7}+C_{520} \lambda X_{2}+C_{550} X_{5}, \\
& f_{6}(\mathbf{X}, \lambda)=C_{615} X_{1} X_{5}+C_{624} X_{2} X_{4}+C_{630} \lambda X_{3}+C_{660} X_{6}, \\
& f_{7}(\mathbf{X}, \lambda)=C_{714} X_{1} X_{4}+C_{725} X_{2} X_{5}+C_{770} X_{7} .
\end{aligned}
$$

It can be verified (Saltzman 1962) that the values of the coefficients $C_{i j k}$ in (2) depend on (i) the fundamental wavelength $L$ in the (horizontal) $x$ and $2 H$ in the (vertical) $z$ directions and (ii) the wavenumbers $m$ and $n$ in the $x$ and $z$ directions, respectively. Table 2 in Saltzman (1962) contains a listing of the numerical values of $C_{i j k}$ for low-order models of order $n \leq 52$. For quick and easy reference, the subset of values of $C_{i j k}$ for the S-LOM (7) in (2) extracted from Saltzman (1962) are given in Table 1 of this paper. Notice that $\lambda$ affects only three components, $f_{4}, f_{5}$, and $f_{6}$, rather directly. Assuming the existence and uniqueness of the solution of (1), let $\boldsymbol{\phi}_{t}[\mathbf{X}(0), \lambda]=\mathbf{X}[t, \mathbf{X}(0), \lambda]$ denote the solution starting from the initial condition $\mathbf{X}(0)$ for a given $\lambda$. Clearly, $\boldsymbol{\phi}_{t}(\cdot, \lambda): R^{7} \rightarrow R^{7}$ defines the nonlinear flow that relates the initial condition $\mathbf{X}(0) \in R^{7}$ to the solution $\mathbf{X}[t, \mathbf{X}(0), \lambda] \in R^{7}$ at time $t \geq 0$. In other words, $\boldsymbol{\phi}_{t}(\cdot, \lambda)$ defines the flow induced by the vector field $\mathbf{f}(\mathbf{X}, \lambda)$ in (1).

Definition 2.1: A subset $\mathbf{S} \subseteq R^{7}$ is an invariant set or subspace for the flow induced by S-LOM (7) in (1) and (2), if, for $\mathbf{X}(0) \in \mathbf{S}, \boldsymbol{\phi}_{t}[\mathbf{X}(0), \lambda] \in \mathbf{S}$ for all $t \geq 0$. That is, the solution starting from $\mathbf{S}$ remains in $\mathbf{S}$ for all times.

Clearly, the whole space $R^{7}$ and the origin are trivially invariant sets. Our interest is in finding nontrivial invariant sets.

Definition 2.2: A set $\mathbf{E} \subset R^{7}$ of points, where the field vanishes, that is,

$$
\mathbf{E}=\left\{(\mathbf{X}, \lambda) \in R^{7} \times R \mid \mathbf{f}(\mathbf{X}, \lambda)=0\right\},
$$

constitutes the equilibria for the S-LOM (7) (Hirsch and Smale 1973).
The equilibria may either be a collection of isolated points or a continuous curve $\mathbf{X}^{*}(\lambda)$ or a manifold in $R^{7}$ parameterized by $\lambda$.

Notice that while an equilibrium is an invariant set, the converse is not true. It turns out that S-LOM (7) enjoys several equilibria and invariant sets.

\section{a. Equilibrium $E_{1}$}

It can be verified by inspection that $\mathbf{X}=0$; the origin of $R^{7}$ is an isolated equilibrium for S-LOM (7) for all $\lambda>0$. That is,

$$
\dot{\mathbf{X}}=\mathbf{f}(0, \lambda)=0 .
$$

\section{b. Invariant subspace}

It turns out that S-LOM (7) in (1) and (2) admits three invariant subspaces labeled as $\mathrm{IS}_{1}, \mathrm{IS}_{2}$, and $\mathrm{IS}_{3}$.

\section{1) INVARIANT SUBSPACE $I_{1}$}

It can be verified that the 3D subspace defined by

$$
\mathrm{IS}_{1}=\left\{X_{2}=X_{3}=X_{5}=X_{6}=0\right\}
$$

is an invariant subspace for S-LOM (7).

By projecting the S-LOM (7) onto this subspace, we obtain the reduced dynamics in $\mathrm{IS}_{1}$ given by

$$
\dot{\mathbf{Y}}=\mathbf{F}(\mathbf{Y}, \lambda),
$$

where $\mathbf{Y}=\left(Y_{1}, Y_{4}, Y_{7}\right)^{\mathrm{T}} \in R^{3}, \mathbf{F}: R^{3} \times R \rightarrow R^{3}$ with $\mathbf{F}=$ $\left(F_{1}, F_{4}, F_{7}\right)^{\mathrm{T}} \in R^{3}$, where 


$$
\begin{aligned}
& F_{1}(\mathbf{Y}, \lambda)=C_{140} Y_{4}+C_{110} Y_{1}, \\
& F_{4}(\mathbf{Y}, \lambda)=C_{417} Y_{1} Y_{7}+C_{410} \lambda Y_{1}+C_{440} Y_{4}, \\
& F_{7}(\mathbf{Y}, \lambda)=C_{714} Y_{1} Y_{4}+C_{770} Y_{7} .
\end{aligned}
$$

This closed subsystem resides in the 3D invariant subspace spanned by the original variables $\left\{X_{1}, X_{4}, X_{7}\right\}$. Notice also that new variables $Y_{i}$ are the surrogates for the original $X_{i}$ for $i=1,4,7$ in IS $_{1}$.

\section{2) INVARIANT SUBSPACE $\mathrm{IS}_{2}$}

Proceeding likewise, it can be verified that

$$
\mathrm{IS}_{2}=\left\{X_{1}=X_{3}=X_{4}=X_{6}=0\right\}
$$

is an invariant subspace for the S-LOM (7) in (1) and (2). Again, by projecting S-LOM (7) onto this subspace, the resulting reduced dynamics in $I S_{2}$ is given by

$$
\dot{\mathbf{Z}}=\mathbf{G}(\mathbf{Z}, \lambda),
$$

where $\mathbf{Z}=\left(Z_{2}, Z_{5}, Z_{7}\right)^{\mathrm{T}} \in R^{3}, \mathbf{G}: R^{3} \times R \rightarrow R^{3}$ with $\mathbf{G}=$ $\left(G_{2}, G_{5}, G_{7}\right)^{\mathrm{T}} \in R^{3}$, where

$$
\begin{aligned}
& G_{2}(\mathbf{Z}, \lambda)=C_{250} Z_{5}+C_{220} Z_{2}, \\
& G_{5}(\mathbf{Z}, \lambda)=C_{527} Z_{2} Z_{7}+C_{520} \lambda Z_{2}+C_{550} Z_{5}, \\
& G_{7}(\mathbf{Z}, \lambda)=C_{725} Z_{2} Z_{5}+C_{770} Z_{7} .
\end{aligned}
$$

This closed subsystem resides in the $3 \mathrm{D}$ invariant subspace spanned by the original variables $\left\{X_{2}, X_{5}, X_{7}\right\}$. Clearly, the new variables $Z_{i}$ are the surrogates for the original $X_{i}$ for $i=2,5,7$ in $\mathrm{IS}_{2}$.

\section{3) INVARIANT SUBSPACE $\mathrm{IS}_{3}$}

The 3D subspace defined by

$$
\mathrm{IS}_{3}=\left\{X_{1}=X_{2}=X_{4}=X_{5}=0\right\}
$$

is an invariant subspace for the S-LOM (7). The projecting S-LOM (7) onto $\mathrm{IS}_{3}$ gives the following linear dynamics:

$$
\dot{\xi}=\mathbf{H} \xi,
$$

where $\boldsymbol{\xi}=\left(\xi_{3}, \xi_{6}, \xi_{7}\right)^{\mathrm{T}} \in R^{3}$ and $\mathbf{H} \in R^{3 \times 3}$ given by

$$
\mathbf{H}=\left[\begin{array}{ccc}
C_{330} & C_{360} & 0 \\
C_{630} \lambda & C_{660} & 0 \\
0 & 0 & C_{770}
\end{array}\right] .
$$

This closed subsystem lies in the 3D invariant subspace spanned by $\left\{X_{3}, X_{6}, X_{7}\right\}$, where $\xi_{i}$ are the surrogates for the original $X_{i}$ in $\mathrm{IS}_{3}$ for $i=3,6,7$.

We hasten to add that a careful reading of the evolution of the solution of the S-LOM (7) given in Fig. 3, page 341 in Saltzman (1962) may suggest the presence of invariant subspaces IS $S_{1}$ and IS $_{2}$.

\section{4) SALTZMAN-LORENZ INTERACTIONS: S-LOM (7)}

We begin this discussion with the key quotation from Lorenz's scientific biography The Essence of Chaos (Lorenz 1993, p. 137) that is most pertinent:

At the Tokyo meeting ${ }^{1}$ more than a decade earlier I had briefly mentioned the unexpected behavior of the twelve-variable model, ${ }^{2}$ but I felt that a full discussion of the relationship between lack of periodicity and growth of small disturbances, and its implications for long-range weather forecasting, belonged in a separate paper. For that paper I was anxious to use an even simpler system of equations as a principal illustrative example... . I tried to simplify the model still more without any luck.... My search came to an abrupt end one afternoon in 1961 when I was visiting Barry Saltzman at the Travelers Research Center... B Barry showed me a system of seven equations that he had been solving numerically. The equations were a bit like mine, but they modeled convective fluid motion driven by heating from below.... He was interested in periodic solutions and had obtained a number of them, but he showed me one solution that refused to settle down. I looked at it eagerly, and noted that four of the seven variables became very small. This suggested that the other three were keeping each other going, so that the system with only these three variables might exhibit the same behavior. Barry gave me the go-ahead signal, and back at M. I. T. the next morning I put the three equations on the computer and, sure enough, there was the same lack of periodicity that Barry had discovered. Here was the long-sought system whose existence I had begun to doubt.

And, of course, exploration of this system led to Lorenz's seminal contribution, deterministic nonperiodic flow (Lorenz 1963).

A number of observations are in order:

1) Lorenz's system of three equations are given by

$$
\begin{aligned}
& \dot{x}=-\sigma x+\sigma y, \\
& \dot{y}=-x z+\rho x-y, \\
& \dot{z}=x y-\beta z .
\end{aligned}
$$

using the spectral expansion (refer to Saltzman 1962, section 7)

\footnotetext{
${ }^{1}$ The International Symposium on Numerical Weather Prediction, Tokyo, Japan, 1960.

${ }^{2}$ A simplified form of the filtered equations of numerical weather forecasting.
} 


$$
\begin{aligned}
& \psi=A \sin \alpha x \sin z \\
& \theta=D \cos \alpha x \sin z+\mathbf{G} \sin z
\end{aligned}
$$

Comparing (10) with the reduced system (5) in $\mathrm{IS}_{1}$ and (7) in $\mathrm{IS}_{2}$, it turns out that both of these are Lorenz-like systems.

2) The three mode models in (5) and (7) differ from the Lorenz model in (10) only in the scaling of the variables. The parameter $\rho$ in (10) corresponds to $\lambda$ in (5) and (7). In the analysis of the Lorenz model (10), the aspect ratio parameter $\beta$ is set at $8 / 3$ and the Prandtl number $\sigma$ is set at 10 . A careful look at the derivation of S-LOM (7) in Saltzman (1962) reveals that he has already incorporated $\beta$ and $\sigma$ in the numerical computation of the values of the coefficients $C_{i j k}$.

3) While our intent is not in analyzing the comparative powers of different low-order models to explain the convection phenomenon, an observation relating to the S-LOM (7) is worth mentioning here.

The second sentence in Saltzman's (1962) conclusions (section 8 of his paper) reads as follows:

...in spite of its simplicity the system treated does, in fact, appear to contain a good deal of the real physical content of the problem [Bénard's laboratory experiment].

To further amplify on this statement, consider water at $20^{\circ} \mathrm{C}$ with the follow physical parameters: coefficient of thermal diffusivity $\kappa=1.5 \times 10^{-3} \mathrm{~cm} \mathrm{~s}^{-2}$, kinematic viscosity $^{3} \nu=1.5 \times 10^{-2} \mathrm{~cm}^{2} \mathrm{~s}^{-1}$, and coefficient of volume expansion $\varepsilon=2.0 \times 10^{-3 \circ} \mathrm{C}^{-1}$, and where the depth of the water $H$ is $2 \mathrm{~mm}$ in accord with order of magnitude fluid depth in experiments reviewed by Chandrasekhar (1961, chapter 2).

In this case, the Rayleigh number given by

$$
R=\frac{g \varepsilon \Delta T H^{3}}{\kappa \nu}=\lambda R_{c}=\lambda \frac{27}{4} \pi^{4},
$$

where $g$ is the acceleration of gravity and $\Delta T$ is the temperature difference over the depth of water. If $\lambda=2$, the adverse temperature gradient is $\Delta T / H \approx 2^{\circ} \mathrm{C} \mathrm{mm}^{-1}$, with $\Delta T=3.7^{\circ} \mathrm{C}$.

Under these circumstances, it is interesting and reasonable to consider the favored convective regime as a function of the Rayleigh number-one with a threewave pattern $(m=3, n=1)$ over the horizontal distance

\footnotetext{
${ }^{3}$ In keeping with Saltzman's model, we keep the Prandtl number $(\operatorname{Pr})=\nu / \kappa=10$ and accordingly change the value of $\nu$ from its true value of $1.0 \times 10^{-2} \mathrm{~cm}^{2} \mathrm{~s}^{-1}$ to the value shown above.
}

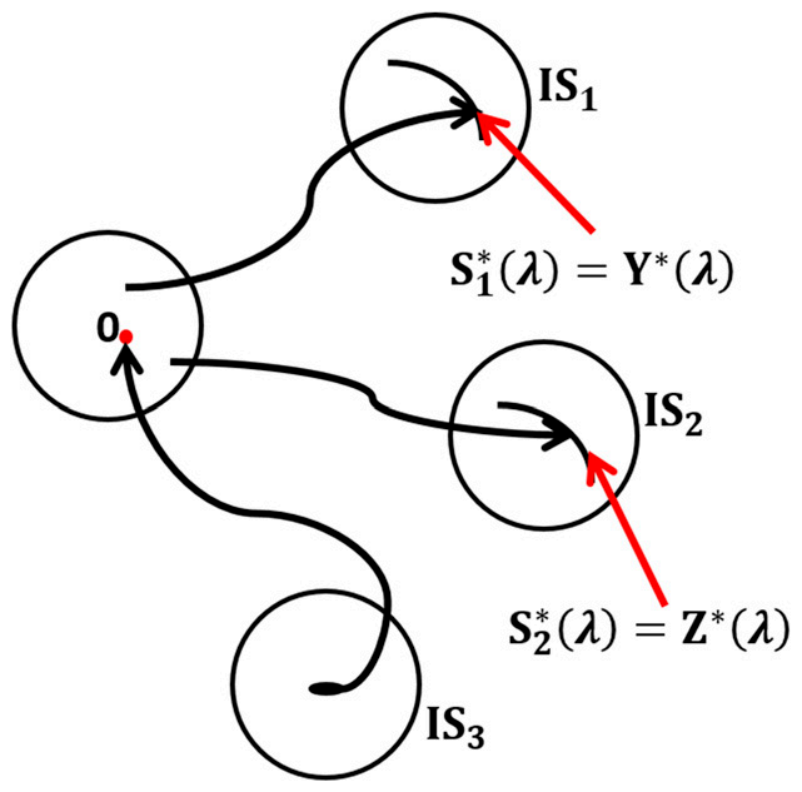

FIG. 1. A pictorial view of the asymptotic behavior of S-LOM (7). While the invariant subspaces $\mathrm{IS}_{1}, \mathrm{IS}_{2}$, and $\mathrm{IS}_{3}$ have a common dimension $X_{7}$, for clarity these invariant subspaces are shown as distinct sets. $S_{1}^{*}(\lambda)$ and $S_{2}^{*}(\lambda)$ are the loci of the stable equilibria embedded in $\mathrm{IS}_{1}$ and $\mathrm{IS}_{2}$, respectively.

of $L=6 \sqrt{2} H$ or four waves over this horizontal distance ( $m=4, n=1)$. The regime at equilibrium for $\lambda=2$ is the 3 -wave regime. If $\lambda$ is decreased by $5 \%, \lambda=1.9$ instead of $\lambda=2$, the three-wave pattern remains with slight changes in magnitude of the spectral components; however, if $\lambda$ is increased by $5 \%$, from $\lambda=2$ to $\lambda=2.1$, the regime changes to a four-wave pattern. In short, if $\Delta T$ is increased from $3.7^{\circ}$ to $3.9^{\circ} \mathrm{C}$, there is a regime change, whereas if it is decreased to $3.5^{\circ} \mathrm{C}$, there is no regime change. In essence, this was the type of question Rayleigh considered in development of theory underlying Bénard's experiments (Rayleigh 1916, 537539) - the size of cells in the presence of fluid instability.

4) S-LOM (7) in (1) and (2) is the union of the three reduced systems in $\mathrm{IS}_{1}, \mathrm{IS}_{2}$, and $\mathrm{IS}_{3}$ and also contains an additional nine nonlinear interaction terms. Despite these additional nonlinear interaction terms, the following analysis shows that asymptotically the solution of S-LOM (7) finds itself in either $\mathrm{IS}_{1}$ or $\mathrm{IS}_{2}$. Further, depending only on the I.C and $0<\lambda \leq$ 24.8046, after entering into $\mathrm{IS}_{1}$ or $\mathrm{IS}_{2}$, the solutions approach stable equilibrium in the $1 \mathrm{D}$ manifold parameterized by $\lambda$. These stable values of the Fourier amplitudes correspond to the periodic solution of the Oberbeck-Boussinesq equation in Saltzman (1962). This property partially captured in Fig. 1. For values of $\lambda>24.8046$, the solution exhibits chaotic behavior in $\mathrm{IS}_{1}$ and $\mathrm{IS}_{2}$. 
TABLE 2. Eigenvalues of $\mathbf{J}_{\mathbf{f}}(\mathbf{X})$ at $\mathbf{X}=0$ for different $\lambda$.

\begin{tabular}{|c|c|c|c|c|c|c|c|}
\hline$\lambda$ & $\alpha_{7}(\lambda)$ & $\alpha_{6}(\lambda)$ & $\alpha_{5}(\lambda)$ & $\alpha_{4}(\lambda)$ & $\alpha_{3}(\lambda)$ & $\alpha_{2}(\lambda)$ & $\alpha_{1}(\lambda)$ \\
\hline 1.0000 & -0.0054 & -1.8751 & -39.4790 & -39.7781 & -162.8456 & -203.1969 & -400.5259 \\
\hline 1.0001 & -0.0040 & -1.8735 & -39.4790 & -39.7781 & -162.8470 & -203.1985 & -400.5259 \\
\hline 1.0002 & -0.0027 & -1.8720 & -39.4790 & -39.7781 & -162.8483 & -203.2000 & -400.5259 \\
\hline 1.0003 & -0.0013 & -1.8704 & -39.4790 & -39.7781 & -162.8497 & -203.2016 & -400.5259 \\
\hline 1.0004 & 0.0000 & -1.8689 & -39.4790 & -39.7780 & -162.8510 & -203.2031 & -400.5260 \\
\hline 1.0005 & 0.0014 & -1.8674 & -39.4790 & -39.7780 & -162.8524 & -203.2046 & -400.5260 \\
\hline 1.0006 & 0.0027 & -1.8658 & -39.4790 & -39.7780 & -162.8537 & -203.2062 & -400.5260 \\
\hline 1.0007 & 0.0040 & -1.8643 & -39.4790 & -39.7780 & -162.8550 & -203.2077 & -400.5260 \\
\hline 1.1225 & 1.6265 & -0.0094 & -39.4790 & -39.7476 & -164.4775 & -205.0626 & -400.5564 \\
\hline 1.1226 & 1.6278 & -0.0078 & -39.4790 & -39.7475 & -164.4788 & -205.0642 & -400.5565 \\
\hline 1.1227 & 1.6291 & -0.0063 & -39.4790 & -39.7475 & -164.4801 & -205.0657 & -400.5565 \\
\hline 1.1228 & 1.6304 & -0.0048 & -39.4790 & -39.7475 & -164.4814 & -205.0672 & -400.5565 \\
\hline 1.1229 & 1.6317 & -0.0033 & -39.4790 & -39.7475 & -164.4827 & -205.0687 & -400.5565 \\
\hline 1.1230 & 1.6331 & -0.0018 & -39.4790 & -39.7474 & -164.4841 & -205.0702 & -400.5566 \\
\hline 1.1231 & 1.6344 & 0.0000 & -39.4790 & -39.7474 & -164.4854 & -205.0717 & -400.5566 \\
\hline 1.1232 & 1.6357 & 0.0012 & -39.4790 & -39.7474 & -164.4867 & -205.0732 & -400.5566 \\
\hline 1.1233 & 1.6370 & 0.0027 & -39.4790 & -39.7474 & -164.4880 & -205.0747 & -400.5566 \\
\hline 1.1234 & 1.6383 & 0.0042 & -39.4790 & -39.7473 & -164.4893 & -205.0762 & -400.5567 \\
\hline 1.1235 & 1.6397 & 0.0057 & -39.4790 & -39.7473 & -164.4907 & -205.0777 & -400.5567 \\
\hline 2 & 12.4903 & 12.4736 & -39.4790 & -39.5286 & -175.3413 & -217.5456 & -400.7754 \\
\hline 10 & 92.3587 & 80.8944 & -37.5448 & -39.4790 & -243.7454 & -297.4307 & -402.7592 \\
\hline 20 & 160.0089 & 138.2498 & -35.0950 & -39.4790 & -301.1008 & -365.0809 & -405.2090 \\
\hline 25 & 187.9856 & 161.9096 & -33.8821 & -39.4790 & -324.7606 & -393.0576 & -406.4219 \\
\hline 30 & 213.4952 & 183.4645 & -32.6771 & -39.4790 & -346.3155 & -407.6269 & -418.5672 \\
\hline
\end{tabular}

\section{Invariant subspaces and equilibria}

Against the backdrop of the existence of equilibria and three invariant subspaces, we now describe the stability of the equilibrium at $E_{1}$ and the asymptotic properties of the three reduced dynamics in $\mathrm{IS}_{i}, i=1,2,3$.

\section{a. Stability of the equilibrium $E_{1}$}

To further characterize the stability properties of $E_{1}$, we need to compute the eigenvalues of the Jacobian, $\mathbf{J}_{\mathbf{f}}(\mathbf{X})$ of the field $\mathbf{f}(\mathbf{X}, \lambda)$ in (2) at $\mathbf{X}=0$ given by

$$
\left.\mathbf{J}_{\mathbf{f}}(\mathbf{X})\right|_{\mathbf{X}=0}=\left[\begin{array}{ccccccc}
C_{110} & 0 & 0 & C_{140} & 0 & 0 & 0 \\
0 & C_{220} & 0 & 0 & C_{250} & 0 & 0 \\
0 & 0 & C_{330} & 0 & 0 & C_{360} & 0 \\
C_{410} \lambda & 0 & 0 & C_{440} & 0 & 0 & 0 \\
0 & C_{520} \lambda & 0 & 0 & C_{550} & 0 & 0 \\
0 & 0 & C_{630} \lambda & 0 & 0 & C_{660} & 0 \\
0 & 0 & 0 & 0 & 0 & 0 & C_{770}
\end{array}\right]
$$

Let $\left\{\alpha_{i}(\lambda), \mathbf{V}_{i}(\lambda)\right\}$ be the eigenvalue-vector pair for the matrix in (12), where it is assumed without loss of generality that $\alpha_{1}(\lambda) \leq \alpha_{2}(\lambda) \leq \cdots \leq \alpha_{7}(\lambda)$. In appendix $\mathrm{A}$, it is shown that this computation of the eigensystem for (12) reduces to that of four separable, simple systems. An immediate consequence of this separability is that it greatly simplifies the problem of computing eigenvectors of (12). It follows from appendix A that exactly two eigenvectors of (12) reside in each of the three invariant subspaces $\mathrm{IS}_{1}, \mathrm{IS}_{2}$, and IS 3 and the seventh one lies along the seventh unit vector $(0,0,0,0,0,0,1)^{\mathrm{T}} \in R^{7}$.
The entries $\alpha_{i}(\lambda), 1 \leq i \leq 7$ of eigenvalues of (12) in Table 2 as a function of $\lambda$ are obtained from the computation in appendix A by sorting the $\mu_{i}(\lambda), 1 \leq i \leq 7$ [ $\mu$ is defined in (A2)-(A6) in appendix A]. For example, $\alpha_{1}(\lambda)=\min _{i}\left\{\mu_{i}(\lambda)\right\}$ and $\alpha_{7}(\lambda)=\max _{i}\left\{\mu_{i}(\lambda)\right\}$.

Referring to Table 2 , the origin ${ }^{i} \mathbf{X}=0$ undergoes bifurcation twice: first at $\lambda_{1}=1.0004$ and again when $\lambda_{2}=1.1231$. It can be verified that the unstable eigenvector $\mathbf{V}_{7}(\lambda)$ at $\lambda=1.0005$ is given by

$$
\mathbf{V}_{7}^{(1)}(\lambda)=(0.0101,0,0,-0.9999,0,0,0)^{\mathrm{T}} \in \mathrm{IS}_{1} .
$$


At $\lambda=2$, the two unstable eigenvectors are given by

$$
\begin{aligned}
& \mathbf{V}_{7}^{(2)}(\lambda)=(0.0093,0,0,-1.000,0,0,0)^{\mathrm{T}} \in \mathrm{IS}_{1}, \\
& \mathbf{V}_{6}^{(2)}(\lambda)=(0,-0.0080,0,0,1.000,0,0)^{\mathrm{T}} \in \mathrm{IS}_{2} .
\end{aligned}
$$

Thus, for $\lambda<1.0004$, the origin is a stable attractor. For $\lambda>1.0004$, the origin becomes a saddle point. For $1.0004<\lambda<1.1231$, solutions starting close to the origin leaves the origin along the unstable direction $\mathbf{V}_{7}^{(1)}(\lambda)$ given above. But for $\lambda>1.1231$, the solutions leave the neighborhood of the origin along $\mathbf{V}_{7}^{(2)}(\lambda)$ or $\mathbf{V}_{6}^{(2)}(\lambda)$ depending on the IC and $\lambda$. Also notice that the energy in $\mathbf{V}_{7}^{(1)}(\lambda)$ and $\mathbf{V}_{7}^{(2)}(\lambda)$ are concentrated along dimensions $X_{1}$ and $X_{4}$ but that for $\mathbf{V}_{6}^{(2)}(\lambda)$ are concentrated along $X_{2}$ and $X_{5}$. It turns out, as shown below, that $\left\{X_{1}, X_{4}\right\}$ and $\left\{X_{2}, X_{5}\right\}$ lie in two complementary invariant subspaces.

We now move on to the analysis of the three reduced subsystems. Since it is easy to analyze the linear subsystem in $\mathrm{IS}_{3}$, we take it up first.

\section{b. Analysis of the reduced linear dynamics in $I S_{3}$}

Referring to (8) and (9), $\xi_{7}$ is decoupled from $\xi_{3}$ and $\xi_{6}$. Besides, since $C_{770}=-39.479$, it turns out that

$$
\xi_{7}(t)=e^{-39.479 t} \xi_{7}(0),
$$

which tends to 0 exponentially fast. The dynamics of $\left(\xi_{3}, \xi_{6}\right)$ is given by

$$
\left(\begin{array}{l}
\dot{\xi}_{3} \\
\dot{\xi}_{6}
\end{array}\right)=\left[\begin{array}{cc}
C_{330} & C_{360} \\
C_{630} \lambda & C_{660}
\end{array}\right]\left(\begin{array}{l}
\xi_{3} \\
\xi_{6}
\end{array}\right) .
$$

The eigenvalues of the $2 \times 2$ matrix in (14) are the roots of

$0=p(\alpha)=\alpha^{2}-\left(C_{330}+C_{660}\right) \alpha+\left[C_{330} C_{660}-C_{360} C_{630} \lambda\right]$.

It can be verified that the two roots $\alpha_{1}$ and $\alpha_{2}$ of $p(\alpha)=$ 0 are real, distinct, and negative for all $\lambda<177.7933$. Hence, the origin $\left(\xi_{3}=0, \xi_{6}=0, \xi_{7}=0\right)$ of the invariant subspace $\mathrm{IS}_{3}$ is a stable attractor in $\mathrm{IS}_{3}$ and $\lim \boldsymbol{\xi}(t)=0$ for all $\boldsymbol{\xi}(0) \in \mathrm{IS}_{3}$. Refer to the illustration in ${ }^{t \rightarrow \infty}$ ig. 1 .

An immediate import of this analysis is that, if any part of the energy in the solution $\mathbf{X}(t)$ of S-LOM (7) starting from initial conditions $\mathbf{X}(0) \notin \mathrm{IS}_{3}$ spills over to $I_{3}$, then that portion of the energy will dissipate to zero.

\section{c. Analysis of stability of the reduced nonlinear dynamics in $I S_{1}$}

Solving the system of three nonlinear equations in the three variables $\left\{Y_{1}, Y_{4}, Y_{7}\right\}$ in

$$
\mathbf{F}(\mathbf{Y})=0,
$$

where $\mathbf{F}(\mathbf{Y})$ is given in (5), we obtain the locus of the equilibrium $\mathbf{Y}^{*}(\lambda)=\mathbf{S}_{1}^{*}(\lambda)$ in $\mathrm{IS}_{1}$ (see Fig. 1 ). To this end, define a new set of parameters:

$$
a_{1}=\frac{C_{110}}{C_{140}}, \quad a_{2}=\frac{C_{714}}{C_{770}}, \quad a_{3}=\frac{C_{417}}{C_{410}}, \quad a_{4}=\frac{C_{440}}{C_{410}} .
$$

The values of $a_{i}$ are given in Table 3 .

Solving (15), it follows that the set of all equilibria of the reduced subsystem (5) in $\mathrm{IS}_{1}$ is given by

$$
\begin{aligned}
& Y_{4}=-a_{1} Y_{1}, \\
& Y_{7}=a_{1} a_{2} Y_{1}^{2},
\end{aligned}
$$

and

$$
a_{1} a_{2} a_{3} Y_{1}^{3}+\left(\lambda-a_{1} a_{4}\right) Y_{1}=0 .
$$

From the third equation, either $Y_{1}=0$ or

$$
a_{1} a_{2} a_{3} Y_{1}^{2}+\left(\lambda-a_{1} a_{4}\right)=0 .
$$

Since $a_{1} a_{2} a_{3}<0$ (see Table 3 ), it follows that

$$
Y_{1}^{2}=\frac{\lambda-a_{1} a_{4}}{\left|a_{1} a_{2} a_{3}\right|}=\frac{\lambda-0.9998}{0.6666} .
$$

Hence, for $\lambda \geq 1, Y_{1}$ is given by

$$
Y_{1}= \pm 1.2248(\lambda-0.9998)^{1 / 2} .
$$

Substituting (18) in the first two equations in (16), we get a total of three equilibria:

$$
\begin{aligned}
\mathbf{Y}^{*} & =(0,0,0)^{\mathrm{T}}, \\
\mathbf{Y}^{*}(\lambda) & =\left( \pm Y_{1}, \mp 98.6973 Y_{1},-69.7889 Y_{1}^{2}\right)^{\mathrm{T}}, \quad \lambda \geq 1 .
\end{aligned}
$$

A remark on the symmetry of the dynamics (4) and (5) is in order here.

It can be verified that the Lorenz-like system (4) and (5) has an intrinsic symmetry with respect to the rotation by $\pi$ radians about the $Y_{7}$ axis. Define

$$
\mathbf{P}=\left[\begin{array}{ccc}
-1 & 0 & 0 \\
0 & -1 & 0 \\
0 & 0 & 1
\end{array}\right]
$$

which represents a rotation operator by $\pi$ radians with respect to the $Y_{7}$ axis. If $\mathbf{Y}=\left(Y_{1}, Y_{4}, Y_{7}\right)^{\mathrm{T}}$, then $\mathbf{P Y}=$ $\left(-Y_{1},-Y_{4}, Y_{7}\right)^{\mathrm{T}}$, and $\mathbf{P}^{2}=\mathbf{I}$, the identity matrix. Also, it can be verified that 
TABLE 3. Values of parameters $a_{i}$ and $b_{i}$, with $i=1,2,3,4$.

\begin{tabular}{lllll}
\hline \hline & & & $i$ & \\
\cline { 2 - 5 } & 1 & 2 & 3 & 4 \\
\hline$a_{i}$ & $C_{110} / C_{140}=98.6973$ & $C_{714} / C_{770}=-0.7071$ & $C_{417} / C_{410}=9.551 \times 10^{-3}$ & $C_{440} / C_{410}=1.013 \times 10^{-2}$ \\
$b_{i}$ & $C_{220} / C_{250}=117.3247$ & $C_{725} / C_{770}=-0.9428$ & $C_{527} / C_{520}=9.551 \times 10^{-3}$ & $C_{550} / C_{520}=9.568 \times 10^{-3}$ \\
\hline
\end{tabular}

$$
\mathbf{P} \dot{\mathbf{Y}}=\mathbf{P F}(\mathbf{Y})=\mathbf{F}(\mathbf{P Y})
$$

That is

$$
\begin{aligned}
& -F_{1}(\mathbf{Y})=F_{1}\left(-Y_{1},-Y_{4}, Y_{7}\right), \\
& -F_{4}(\mathbf{Y})=F_{4}\left(-Y_{1},-Y_{4}, Y_{7}\right), \\
& +F_{7}(\mathbf{Y})=F_{7}\left(-Y_{1},-Y_{4}, Y_{7}\right) .
\end{aligned}
$$

Stated in other words, (4) and (5) are said to be equivariant (Gilmore and Letellier 2007) under the symmetry matrix $\mathbf{P}$. Hence, it is not surprising that there is a system of two families of equilibria with signs given by $(+,-,-)^{\mathrm{T}}$ and $(-,+,-)^{\mathrm{T}}$, which are rotations by $\pi$ radians of each other with respect to $Y_{7}$. For future reference, we denote these two branches of the equilibrium manifold as $\operatorname{IS}_{1}(+,-,-)$ and $\operatorname{IS}_{1}(-,+,-)$, respectively.

To get an idea of the structure of this equilibria, from (18) and (19), consider one of the branches given by

$$
\begin{aligned}
& Y_{1}^{*}(\lambda)=1.2248(\lambda-0.9998)^{1 / 2}, \\
& Y_{4}^{*}(\lambda)=-a_{1} Y_{1}^{*}(\lambda)=-98.6973 Y_{1}^{*}(\lambda), \\
& Y_{7}^{*}(\lambda)=a_{1} a_{2}\left[Y_{1}^{*}(\lambda)\right]^{2}=-69.7889\left[Y_{1}^{*}(\lambda)\right]^{2} .
\end{aligned}
$$

A plot of $Y_{i}^{*}(\lambda)$ versus $\lambda$ and a 3D view of this branch of equilibria for $0<\lambda \leq 30$ are given in Fig. 2 and Fig. 3.

A sampling of the steady-state values as a function of $\lambda$ are given in Table 4 .

To characterize the stability of these equilibria, first consider the Jacobian of (4) and (5) given by

$$
\mathbf{J}_{\mathbf{F}}(\mathbf{Y})=\left[\begin{array}{ccc}
C_{110} & C_{140} & 0 \\
C_{417} Y_{7}+C_{410} \lambda & C_{440} & C_{417} Y_{1} \\
C_{714} Y_{4} & C_{714} Y_{1} & C_{770}
\end{array}\right]
$$

\section{1) Analysis of the equilibrium at $\mathbf{Y}^{*}=0$}

At this equilibrium, $\mathbf{J}_{\mathbf{F}}(\mathbf{Y})$ takes the form

$$
\mathbf{J}_{\mathbf{F}}(0)=\left[\begin{array}{ccc}
C_{110} & C_{140} & 0 \\
C_{410} \lambda & C_{440} & 0 \\
0 & 0 & C_{770}
\end{array}\right],
$$

whose eigenvalues are given by $\alpha_{3}=C_{770}=-39.479<0$ and the two roots of

$p(\alpha)=\alpha^{2}-\left(C_{110}+C_{440}\right) \alpha+\left[C_{110} C_{440}-C_{140} C_{410} \lambda\right]=0$.

It can be verified the two roots of (23) are real and negative only for $\lambda<0.9999$. Hence, this equilibrium in $\operatorname{IS}_{1}(+,-,-)$ is unstable for $\lambda \geq 1$.

\section{2) ANALysis of THE EQUiLIBRIUM AT $\mathbf{Y}^{*}(\lambda)$}

The variation of the equilibrium $\mathbf{Y}=\left(Y_{1}, Y_{4}, Y_{7}\right)$ and the eigenvalues of the Jacobian at the equilibria of the Lorenz-like system in (4) and (5) for $0<\lambda \leq 30$ are given in Table 4. For $0<\lambda \leq 24.8096$, this equilibrium is an attractor and the solution $\mathbf{Y}(t)$ spiral toward and settle down on $\mathbf{Y}$ listed in Table 4. Recall that $Y_{1}=X_{1}, Y_{4}=X_{4}$, and $Y_{7}=X_{7}$ are the three amplitudes of the 2D Fourier expansion of the solution of the
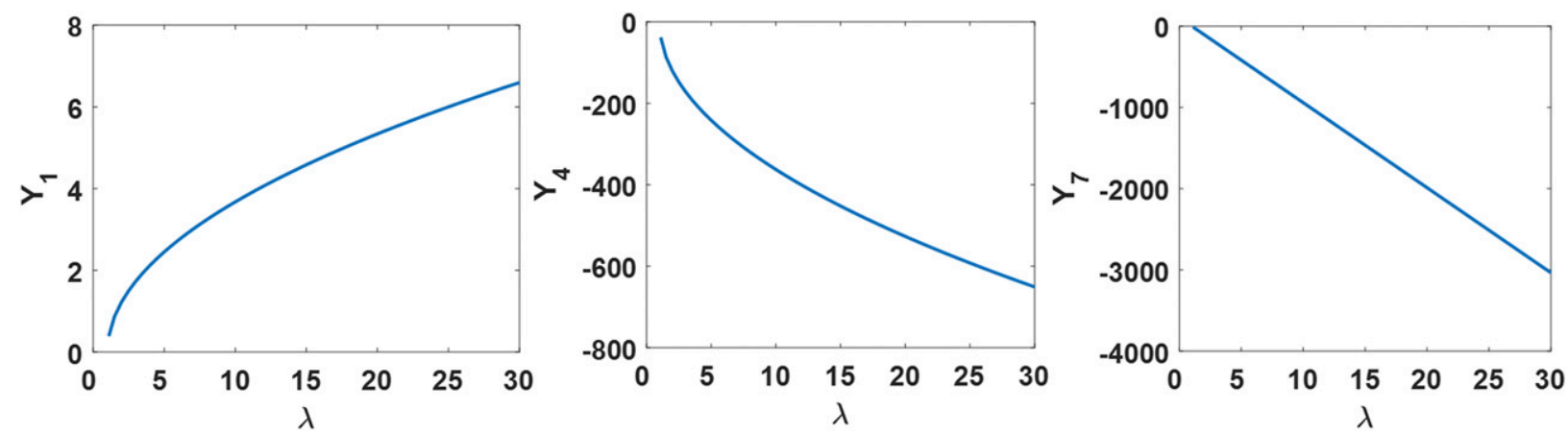

FIG. 2. Plots of the steady states $\left(Y_{1}, Y_{4}, Y_{7}\right)$ in $\mathrm{IS}_{1}(+,-,-)$ as a function of $\lambda$. 


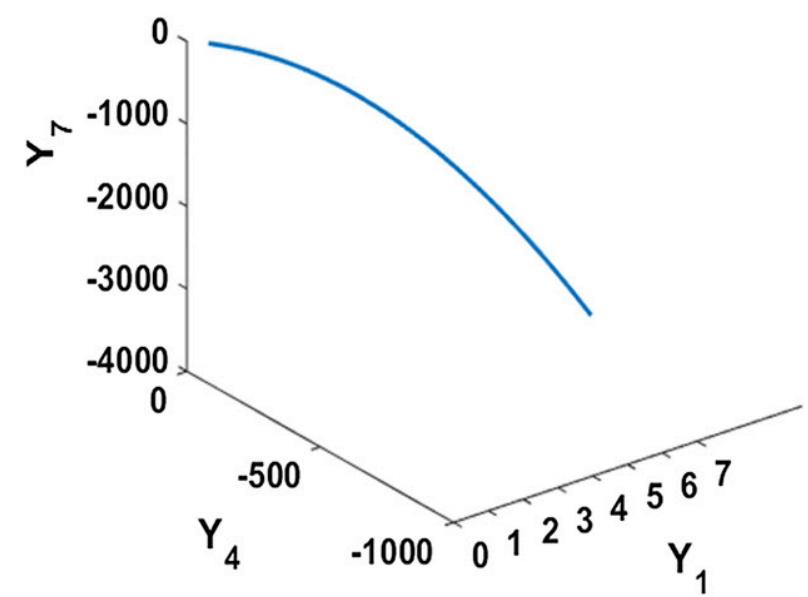

FIG. 3. A 3D plot of the steady states in $\operatorname{IS}_{1}(+,-,-)$ as $\lambda$ is varied from 1.1 to 30

Oberbeck-Boussinesq approximation (Saltzman 1962). Thus, for $\lambda$ in this range, the stable asymptotic solution $\mathbf{Y}=\left(Y_{1}, Y_{4}, Y_{7}\right)$ naturally corresponds to the periodic solution in the physical space. However, for $\lambda>24.8096$, the solution of (4) and (5) undergoes Hopf bifurcation, the equilibria becomes unstable, and the solution in $\mathrm{IS}_{1}$ exhibits chaotic behavior. Stated in other words, the Lorenz-like system in (4) and (5) exhibits characteristics quite similar to the Lorenz (1963) model in (10). Refer to chapter 32 in Lewis et al. (2006) for details.

A sample of this chaotic behavior for $\lambda=28$ and IC $Y_{1}=0, Y_{4}=1.0$, and $Y_{7}=0$ is illustrated in Fig. 4a. We further quantify the key signatures of this chaotic behavior by computing the Lyapunov exponent (Wolf et al. 1985) and the fractal dimension (Grassberger and Procaccia, 1983) of the strange attractor for the system (4) and (5) in $I_{1}$. Variation of the Lyapunov index with $\lambda$ is given in Fig. 4b. Clearly, $L_{1}$ becomes positive when $\lambda \approx 24.8096$, indicating the onset of instability leading to the chaotic behavior. Further, the sum $L_{1}+L_{2}+L_{3}<0$ for all $\lambda$ confirms that the phase volume shrinks even in this chaotic regime. A comparison of the fractal dimension of the strange attractor for the Lorenz model in (10), the Lorenz-like system (4) and (5) in $\mathrm{IS}_{1}$, and (6) and (7) in $\mathrm{IS}_{2}$ are given in Table 5.

\section{d. Analysis of asymptotic stability of the reduced nonlinear dynamics in $\mathrm{IS}_{2}$}

Solving the system of three nonlinear equations in the three variables in $\left\{Z_{2}, Z_{5}, Z_{7}\right\}$ in

$$
\mathbf{G}(\mathbf{Z})=0,
$$

where $\mathbf{G}(\mathbf{Z})$ is given in (7), we obtain the locus of the equilibria $\mathbf{Z}^{*}(\lambda)=S_{2}^{*}(\lambda)$ in $\mathrm{IS}_{2}$ (see Fig. 1). To this end, define control parameters

$b_{1}=\frac{C_{220}}{C_{250}}, \quad b_{2}=\frac{C_{725}}{C_{770}}, \quad b_{3}=\frac{C_{527}}{C_{520}}, \quad b_{4}=\frac{C_{550}}{C_{520}}$.

The values of $b_{i}$ are given in Table 3 .

Solving (24), it follows that the set of all equilibria for the reduced dynamics in (6) and (7) in $\mathrm{IS}_{2}$ are given by

$$
\begin{aligned}
Z_{5} & =-b_{1} Z_{2}, \\
Z_{7} & =b_{1} b_{2} Z_{2}^{2}, \\
b_{1} b_{2} b_{3} Z_{2}^{3}+\left(\lambda-b_{1} b_{4}\right) Z_{2} & =0 .
\end{aligned}
$$

From the third equation in (26), either $Z_{2}=0$ or

TABLE 4. A sampling of the values of the steady states in $\mathrm{IS}_{1}(+,-,-)$ as a function of $\lambda$.

\begin{tabular}{ccccccc}
\hline \hline$\lambda$ & $Y_{1}$ & $Y_{4}$ & $Y_{7}$ & $E V_{1}$ & $E V_{2}$ & $E V_{3}$ \\
\hline 1.1000 & 0.3877 & -38.2652 & -10.4902 & -163.2276 & -36.15430 & -2.9481 \\
1.5000 & 0.8662 & -85.4953 & -52.3673 & -164.7026 & $-18.8137+13.1189 i$ & $-18.8137-13.1189 i$ \\
2.0000 & 1.2249 & -120.8965 & -104.7137 & -166.4253 & $-17.9523+26.8035 i$ & $-17.9523-26.8035 i$ \\
5.0000 & 2.4497 & -241.7750 & -418.7920 & -174.8082 & $-13.7609+61.4196 i$ & $-13.7609-61.4196 i$ \\
10.0000 & 3.6744 & -362.6574 & -942.2559 & -184.6612 & $-8.8344+91.4311 i$ & $-8.8344-91.4311 i$ \\
15.0000 & 4.5828 & -452.3114 & -1465.7198 & -191.9135 & $-5.2083+112.2583 i$ & $-5.2083-112.2583 i$ \\
20.0000 & 5.3388 & -526.9259 & -1989.1837 & -197.6898 & $-2.3201+128.9694 i$ & $-2.3201-128.9694 i$ \\
24.0000 & 5.8740 & -579.7440 & -2407.9547 & -201.5993 & $-0.3654+140.5365 i$ & $-0.3654-140.5365 i$ \\
24.5000 & 5.9375 & -586.0116 & -2460.3011 & -202.0528 & $-0.1386+141.8967 i$ & $-0.1386-141.8967 i$ \\
24.8096 & 5.9764 & -589.8592 & -2492.7140 & -202.3300 & $0.0000+142.7305 i$ & $0.0000-142.7305 i$ \\
25.0000 & 6.0003 & -592.2129 & -2512.6475 & -202.4992 & $0.0846+143.2402 i$ & $0.0846-143.2402 i$ \\
26.0000 & 6.1240 & -604.4247 & -2617.3403 & -203.3720 & $0.5210+145.8789 i$ & $0.5210-145.8789 i$ \\
27.0000 & 6.2453 & -616.3946 & -2722.0331 & -204.2191 & $0.9445+148.4569 i$ & $0.9445-148.4569 i$ \\
28.0000 & 6.3643 & -628.1364 & -2826.7258 & -205.0420 & $1.3560+150.9780 i$ & $1.3560-150.9780 i$ \\
29.0000 & 6.4811 & -639.6627 & -2931.4186 & -205.8421 & $1.7561+153.4454 i$ & $1.7561-153.4454 i$ \\
30.0000 & 6.5958 & -650.9849 & -3036.1114 & -206.6206 & $2.1453+155.8624 i$ & $2.1453-155.8624 i$ \\
\hline
\end{tabular}


(a)
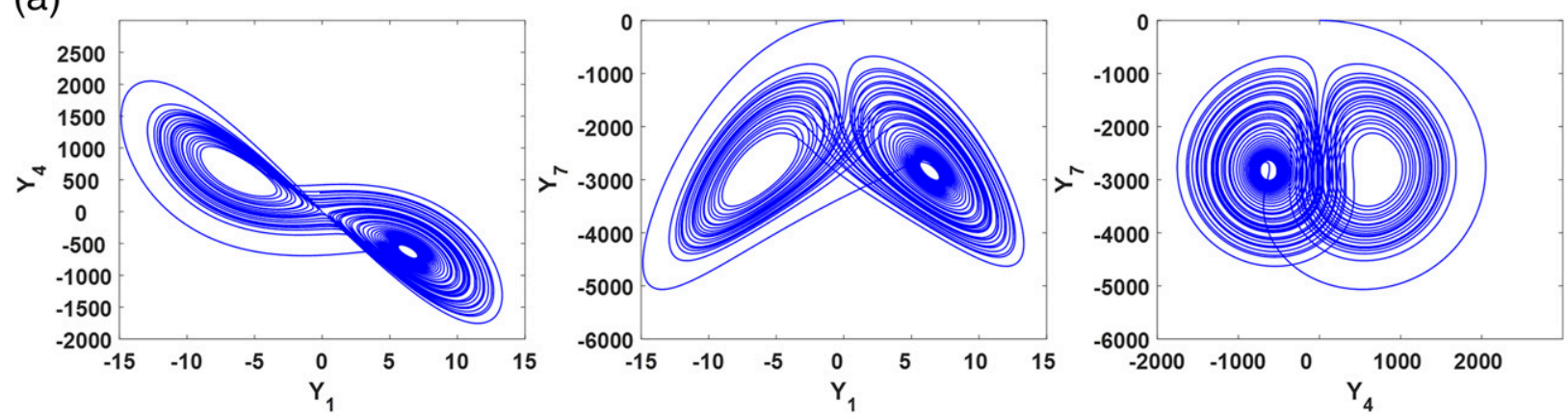

(b)
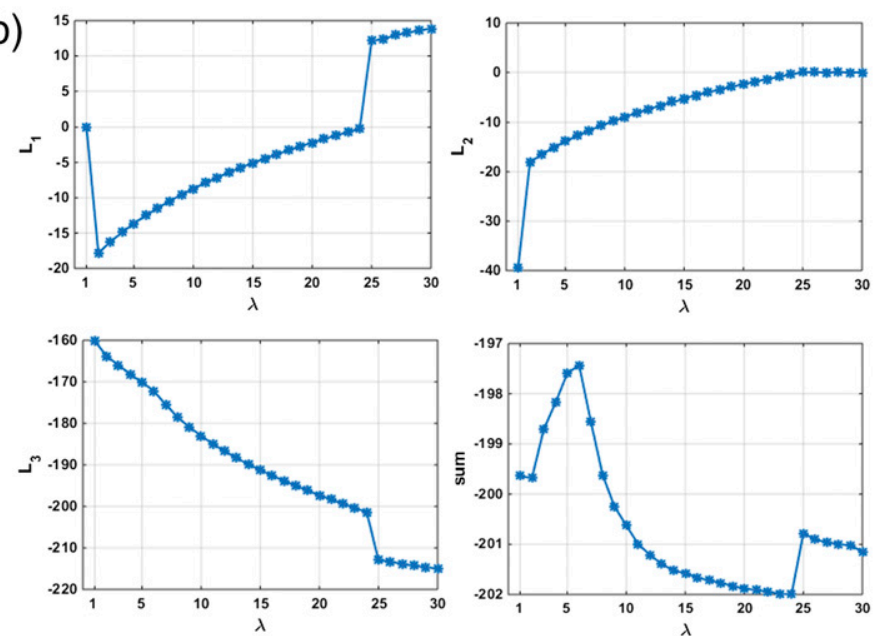

FIG. 4. (a) Phase plot for chaotic behavior in $\mathrm{IS}_{1}\left[\mathrm{IC}, Y_{1}(0)=0, Y_{4}(0)=1, Y_{7}(0)=0\right.$, and $\left.\lambda=28\right]$. (b) Variation of the Lyapunov exponent with $\lambda$ for the Lorenz-like dynamics (4) and (5) in IS1 starting from the same initial condition as in Fig. 4a, $L_{i}$ refers to the $i$ th exponent and sum is $\sum_{i=1}^{3} L_{i}$.

$$
b_{1} b_{2} b_{3} Z_{2}^{2}+\left(\lambda-b_{1} b_{4}\right)=0
$$

Since $b_{1} b_{2} b_{3}$ is negative (refer to Table 3 ), it is immediate that

$$
Z_{2}^{2}=\frac{\lambda-b_{1} b_{4}}{\left|b_{1} b_{2} b_{3}\right|}=\frac{\lambda-1.1226}{1.0565}
$$

Hence, for $\lambda>1.1226, Z_{2}$ is given by

$$
Z_{2}= \pm 0.9729(\lambda-1.1226)^{1 / 2}
$$

Substituting (28) in the first two equations in (26), we obtain a total of three equilibria given by

$$
\begin{aligned}
\mathbf{Z}^{*} & =(0,0,0)^{\mathrm{T}}, \\
\mathbf{Z}^{*}(\lambda) & =\left( \pm Z_{2}, \mp Z_{5}, Z_{7}\right)^{\mathrm{T}}, \quad \lambda \geq 1 .
\end{aligned}
$$

For future reference, we denote these two branches of the equilibrium manifold in $\operatorname{IS}_{2}$ as $\operatorname{IS}_{2}(+,-,-)$ and $\mathrm{IS}_{2}(-,+,-)$, respectively.

To analyze the stability of these equilibria, consider the Jacobian of (6) and (7) given by

$$
\mathbf{J}_{\mathbf{G}}(\mathbf{Z})=\left[\begin{array}{ccc}
C_{220} & C_{250} & 0 \\
C_{527} Z_{7}+C_{520} \lambda & C_{550} & C_{527} Z_{2} \\
C_{725} Z_{5} & C_{725} Z_{2} & C_{770}
\end{array}\right]
$$

TABLE 5. Comparison of the fractal dimension of Lorenz attractor with those of the three sets of attractors arising from S-LOM (7).

\begin{tabular}{ccccccc}
\hline \hline & & \multicolumn{3}{c}{$\lambda$} \\
\cline { 3 - 7 } Name of the attractor & Projection & 25 & 30 & 35 & 40 & 45 \\
\hline Lorenz & 3D & 2.053 & 2.064 & 2.071 & 2.076 & 2.081 \\
Attractor in IS & 3D & 2.057 & 2.065 & 2.072 & 2.077 & 2.082 \\
Attractor in IS $_{2}$ & 3D & 2.051 & 2.059 & 2.064 & 2.069 & 2.074 \\
\hline
\end{tabular}



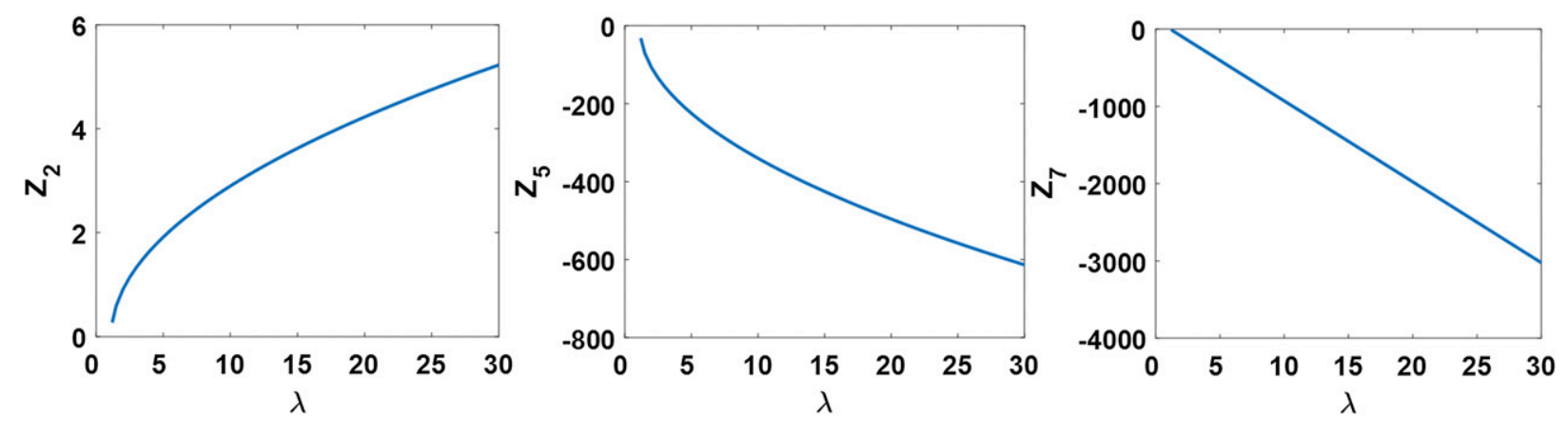

FIG. 5. Plots of the steady states $\left(Z_{2}, Z_{5}, Z_{7}\right)$ in $\mathrm{IS}_{2}(+,-,-)$ as a function of $\lambda$.

\section{1) ANALysis of THE EQUiLIBRIUM At $\mathbf{Z}^{*}=0$}

At this equilibrium, $\mathbf{J}_{\mathbf{G}}(\mathbf{Z})$ takes the form

$$
\mathbf{J}_{\mathbf{G}}(0)=\left[\begin{array}{ccc}
C_{220} & C_{250} & 0 \\
C_{520} \lambda & C_{550} & 0 \\
0 & 0 & C_{770}
\end{array}\right],
$$

whose eigenvalues are given by $\alpha_{3}=C_{770}=-39.479<0$ and the two roots of

$p(\alpha)=\alpha^{2}-\left(C_{220}+C_{550}\right) \alpha+\left[C_{220} C_{550}-C_{250} C_{520} \lambda\right]=0$.

It can be verified the two roots of (33) are real and negative only for $\lambda<1.1226$. Hence, this equilibrium is unstable for $\lambda>1.1226$.

Since (6) and (7) are also a Lorenz-like system, it enjoys symmetry under rotation by $\pi$ radians. Hence there are two branches of the equilibria with signs $\left(+,-,{ }^{*}\right)^{\mathrm{T}}$ and $(-,+, *)^{\mathrm{T}}$.

\section{2) ANALysis of THE EQUILIBRIUM AT $\mathbf{Z}^{*}(\lambda)$}

For definiteness, consider the branch of equilibria given by

$$
\begin{aligned}
& Z_{2}^{*}(\lambda)=0.9729(\lambda-1.1226)^{1 / 2} \\
& Z_{5}^{*}(\lambda)=-b_{1} Z_{2}^{*}(\lambda)=-117.3247 Z_{2}^{*}(\lambda), \\
& Z_{7}^{*}(\lambda)=b_{1} b_{2}\left[Z_{2}^{*}(\lambda)\right]^{2}=-110.6137\left[Z_{2}^{*}(\lambda)\right]^{2} .
\end{aligned}
$$

A plot of the steady states $Z_{2}, Z_{5}, Z_{7}$ versus $\lambda$ are given in Fig. 5. Figure 6 contains a 3D plot of the steady states, which in turn defines periodic solution in the physical space. A sampling of the values of the steady states in $\mathrm{IS}_{2}$ and the eigenvalues of the Jacobian along the steady states for $0<\lambda \leq 30$ are given in Table 6. Again, the solution of (6) and (7) undergoes a Hopf bifurcation at $\lambda^{*}=24.7498$, and for $\lambda>\lambda^{*}$, the equilibria become unstable. In this unstable regime, the model exhibits chaotic behavior. Fractal dimension of the resulting attractor is given in Table 5. A sample of this behavior for $\lambda=28$ and IC $Z_{2}(0)=0.01, Z_{5}(0)=0.01$, and $Z_{7}(0)=0.01$ is illustrated in Fig. 7 .

\section{A global perspective}

In this section, we address two important global properties of the S-LOM (7) in (1). These relate to the rate of change of phase volume and the boundedness of the trajectories of (1).

The divergence of the vector field $\mathbf{f}(\mathbf{X}, \lambda)$ in (1) using the values of the coefficients in Table 1 is given by

$$
D_{\mathbf{X}} \mathbf{f}(\mathbf{X}, \lambda)=\sum_{i=1}^{7} \frac{\partial f_{i}}{\partial X_{i}}=\sum_{i=1}^{7} C_{i i 0}<0 .
$$

Hence, by Liouville's theorem in Arnold (1992, section 6 , article 27 , chapter 3 ), the phase volume decreases at an exponential rate proportional to the term $\sum_{i=1}^{7}\left|C_{i i 0}\right|$. Consequently, the equilibrium has to be a manifold of zero phase volume. This is corroborated by our analysis in sections 2 and 3 , where it is shown that the equilibrium is either the origin or the $1 \mathrm{D}$ manifolds in $\mathrm{IS}_{1}$ and $\mathrm{IS}_{2}$.

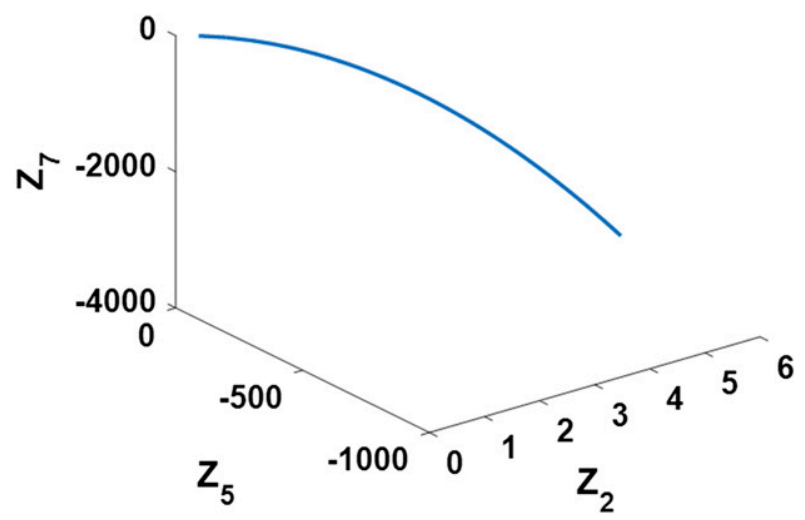

FIG. 6. A 3D plot of the steady states in $\mathrm{IS}_{2}(+,-,-)$ as $\lambda$ is varied from 1.2 to 30 . 
TABLE 6. A sampling of the values of the steady states in $\mathrm{IS}_{2}(+,-,-)$ as $\lambda$ is varied.

\begin{tabular}{ccccccc}
\hline \hline$\lambda$ & $Z_{2}$ & $Z_{5}$ & $Z_{7}$ & $E V_{1}$ & $E V_{2}$ & $E V_{3}$ \\
\hline 1.2000 & 0.2707 & -31.7562 & -8.1038 & -205.3133 & -36.7189 & -2.5188 \\
1.5000 & 0.5977 & -70.1227 & -39.5137 & -206.2597 & $-19.1456+9.0085 i$ & $-19.1456+9.0085 i$ \\
2.0000 & 0.9113 & -106.9194 & -91.8635 & -207.7737 & $-18.3887+26.3576 i$ & $-18.3887-26.3576 i$ \\
5.0000 & 1.9157 & -224.7646 & -405.9625 & -215.5706 & $-14.4902+64.7177 i$ & $-14.4902-64.7177 i$ \\
10.0000 & 2.8988 & -340.0952 & -929.4609 & -225.4637 & $-9.5437+97.6565 i$ & $-9.5437-97.6565 i$ \\
15.0000 & 3.6243 & -425.2181 & -1452.9592 & -233.1334 & $-5.7088+120.5101 i$ & $-5.7088-120.5101 i$ \\
20.0000 & 4.2271 & -495.9396 & -1976.4576 & -239.4364 & $-2.5573+138.8224 i$ & $-2.5573-138.8224 i$ \\
24.0000 & 4.6534 & -545.9602 & -2395.2563 & -243.7899 & $-0.3805+151.4785 i$ & $-0.3805-151.4785 i$ \\
24.7498 & 4.7040 & -551.8941 & -2447.6061 & -244.2993 & $-0.1259+152.9656 i$ & $-0.1259-152.9656 i$ \\
24.5000 & 4.7291 & -554.8349 & -2473.7601 & -244.5511 & $0.0000+153.7015 i$ & $0.0000-153.7015 i$ \\
25.0000 & 4.7540 & -557.7649 & -2499.9560 & -244.8015 & $0.1253+154.4340 i$ & $0.1253-154.4340 i$ \\
26.0000 & 4.8526 & -569.3249 & -2604.6557 & -245.7859 & $0.6174+157.3176 i$ & $0.6174-157.3176 i$ \\
27.0000 & 4.9491 & -580.6547 & -2709.3553 & -246.7444 & $1.0967+160.1338 i$ & $1.0967-160.1338 i$ \\
28.0000 & 5.0438 & -591.7677 & -2814.0550 & -247.6784 & $1.5637+162.8869 i$ & $1.5637-162.8869 i$ \\
29.0000 & 5.1368 & -602.6759 & -2918.7547 & -248.5892 & $2.0191+165.5805 i$ & $2.0191-165.5805 i$ \\
30.0000 & 5.2281 & -613.3900 & -3023.4543 & -249.4778 & $2.4634+168.2181 i$ & $2.4634-168.2181 i$ \\
\hline
\end{tabular}

To examine the boundedness of the solution of (1), consider a generalized energy functional given by

$$
E(\mathbf{X})=\frac{1}{2} \mathbf{X}^{\mathrm{T}} \mathbf{K X}
$$

where $\mathbf{K}$ is a diagonal matrix given by

$$
\mathbf{K}=\operatorname{Diag}\left(K_{1}, K_{2}, \ldots, K_{7}\right),
$$

with $K_{i}>0$ for $1 \leq i \leq 7$.

It is shown in appendix B that for large time $t$,

$$
\dot{E}(\mathbf{X})<0 .
$$

Since the vector field in (1) is smooth in time $t$, the solution $\mathbf{X}(t)$ cannot become unbounded in finite time. Combining these, it is immediate that the solution $\mathbf{X}(t)$ remains bounded for all time $t \geq 0$.

\section{An ensemble analysis}

To further understand the dependence of the behavior of the solution of (1) on the initial conditions and the parameter $\lambda$, in this section, we describe the results of a
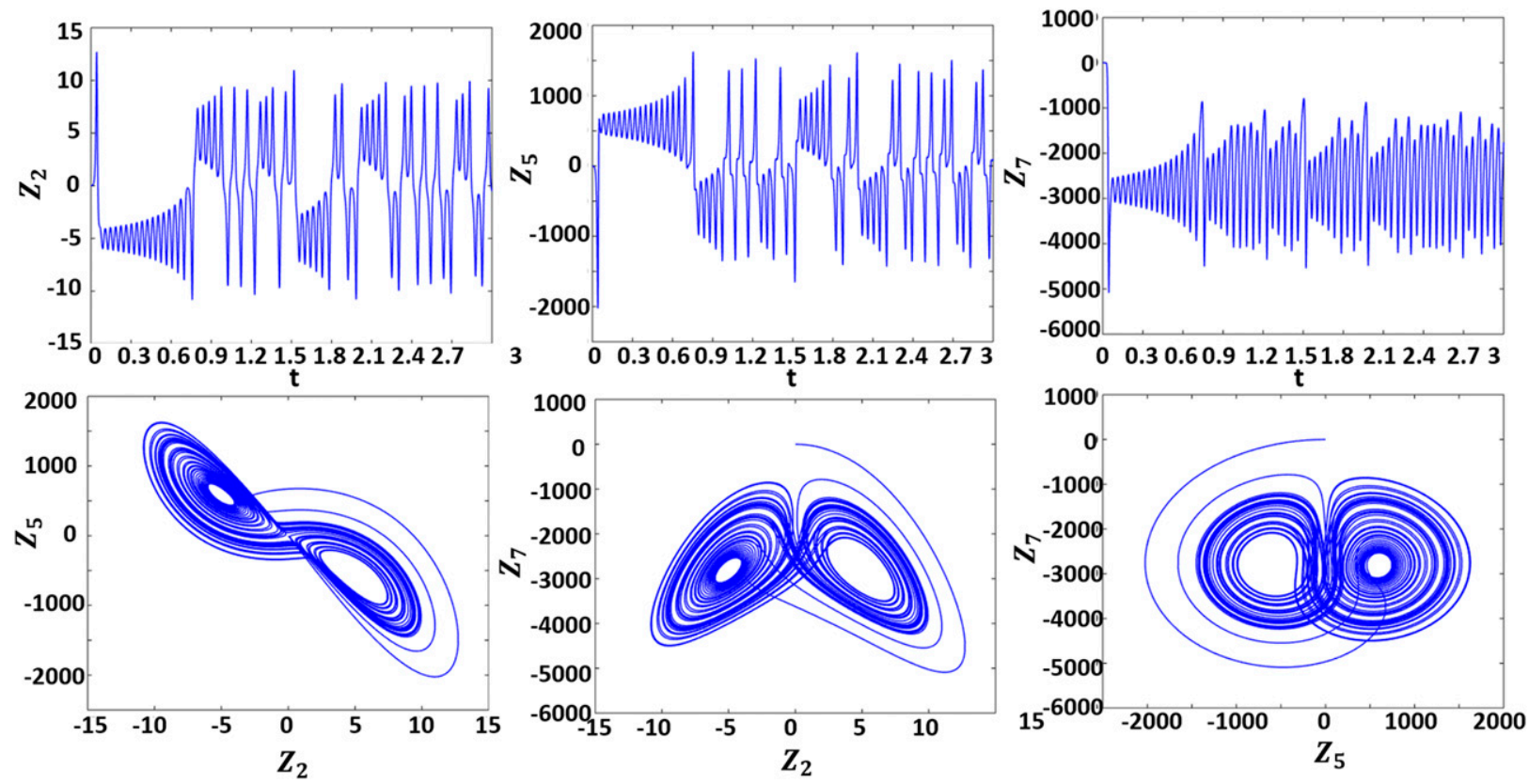

FIG. 7. Time evolution and phase plot for chaotic behavior in IS2 [IC, $Z_{2}(0)=0.01, Z_{5}(0)=0.01, Z_{7}(0)=0.01$ and $\left.\lambda=28\right]$. 


$$
\mathrm{IS}_{1}(+,-,-)
$$
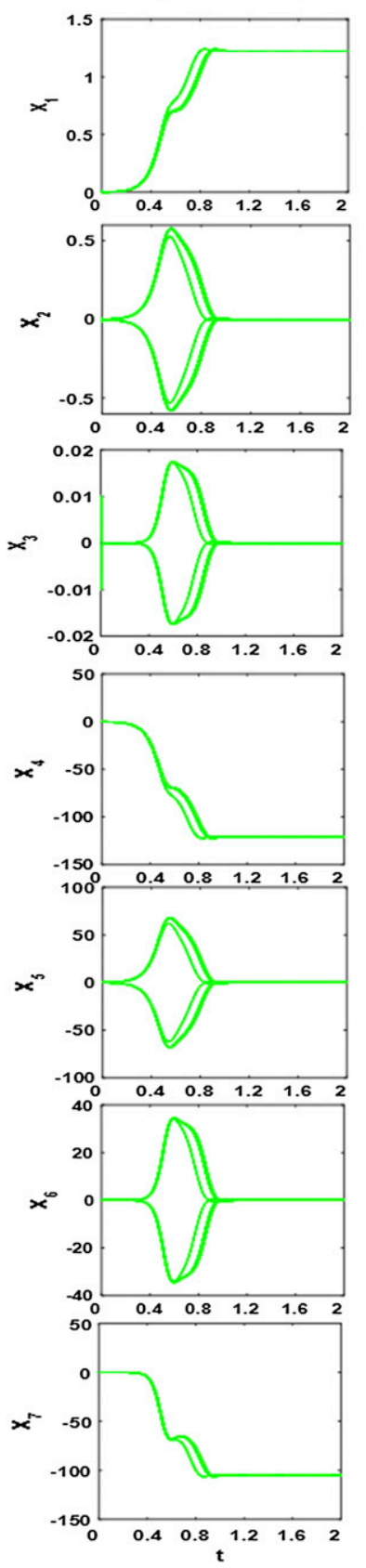

FIG. 8. Plot of the components of the solution of (1) for $\lambda=2$ converging to equilibria in $\mathrm{IS}_{1}$.

deterministic ensemble experiment. We choose a set of $2^{7}=128$ initial conditions corresponding to the coordinates of the vertices/corners of the seven-dimensional hypercube centered at the origin with the length of the sides given by $2 \alpha$ for $\alpha \in(0,1]$ and varying $\lambda$. Thus, $(-\alpha,-\alpha, \ldots,-\alpha)^{\mathrm{T}}$ is one vertex as is $(\alpha, \alpha, \ldots, \alpha)^{\mathrm{T}}$. Using the one-to-one encoding scheme described in
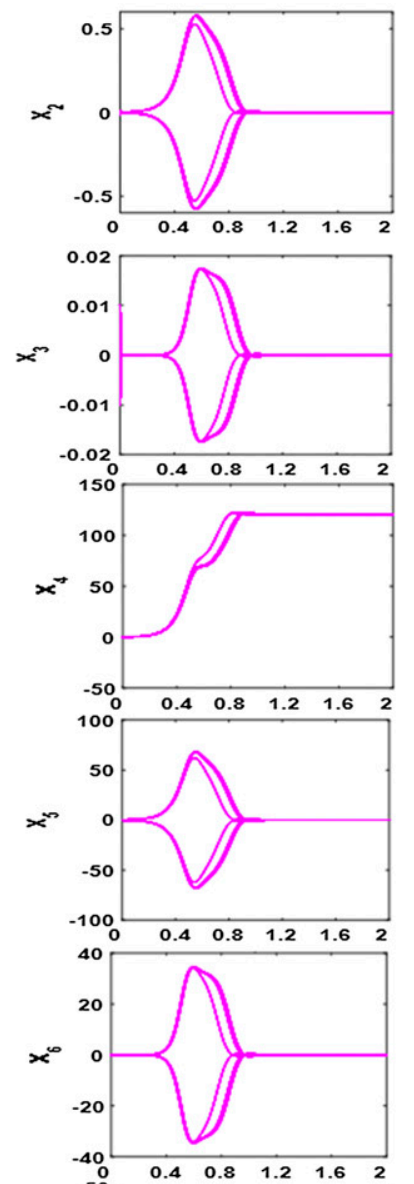
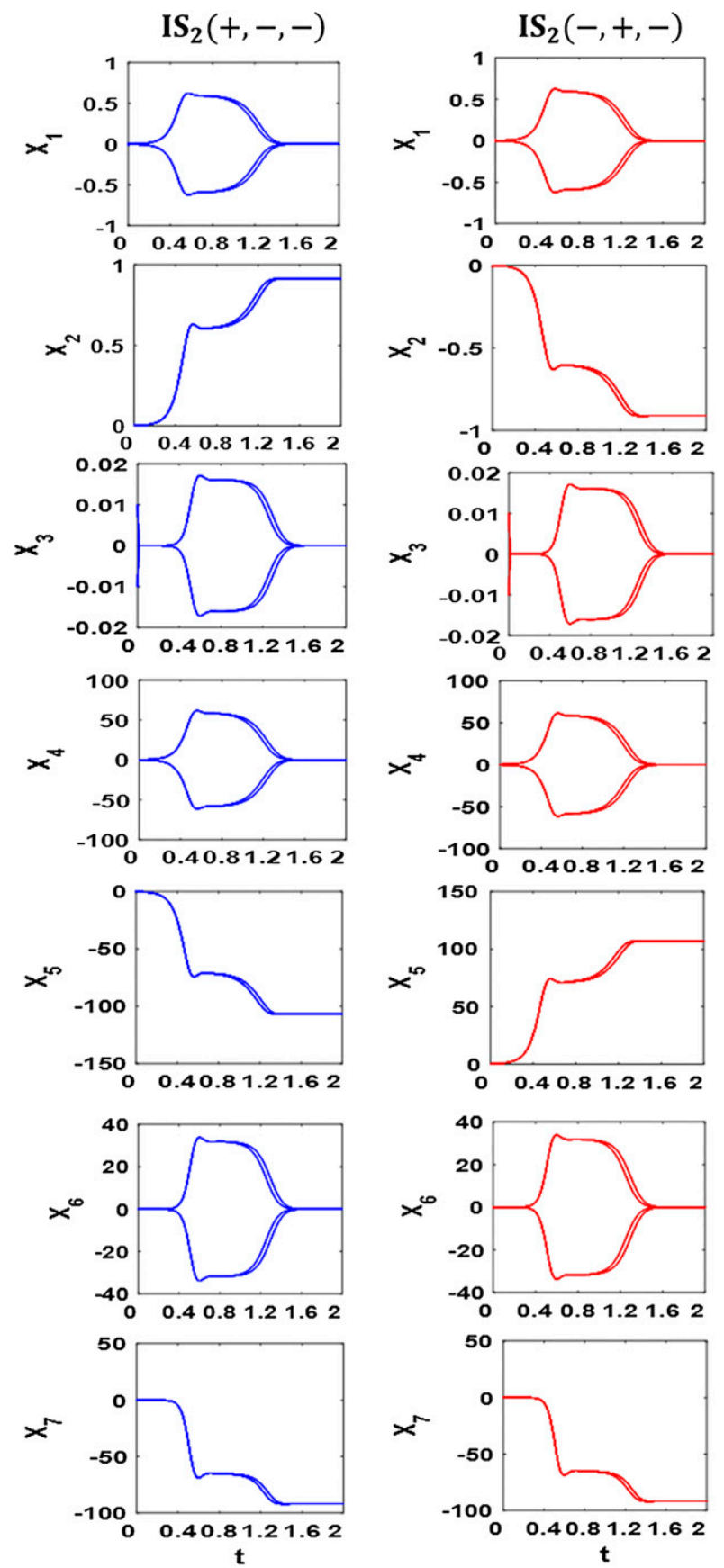

FIG. 9. Plot of the solution of (1) for $\lambda=2$ converging to equilibria in $\mathrm{IS}_{2}$.

appendix $\mathrm{C}$, we compress the seven coordinates into an integer. Accordingly, the vertex with coordinates $(-\alpha,-\alpha, \ldots,-\alpha)^{\mathrm{T}}$ is denoted by the number zero and the vector with coordinates $(\alpha, \alpha, \ldots, \alpha)^{\mathrm{T}}$ is denoted by the number 127. Likewise, the vector with coordinate $(\alpha,-\alpha, \alpha,-\alpha,-\alpha, \alpha, \alpha)^{\mathrm{T}}$ is denoted by the integer 83 . See appendix $\mathrm{C}$ for details. 
TABLE 7. A record of the equilibria to which the solutions converge when $\lambda=2$.

\begin{tabular}{|c|c|c|c|c|c|c|c|c|c|c|c|c|c|c|c|c|}
\hline \multirow{2}{*}{$\frac{\text { Equilibrium }}{\mathrm{IS}_{1}(+,-,-)}$} & \multicolumn{16}{|c|}{ Encoding of initial conditions } \\
\hline & 1 & 3 & 5 & 7 & 9 & 13 & 17 & 19 & 21 & 23 & 27 & 31 & 33 & 35 & 37 & 39 \\
\hline \multirow{5}{*}{$\mathrm{IS}_{1}(-,+,-)$} & 41 & 45 & 49 & 51 & 53 & 55 & 59 & 63 & 65 & 67 & 69 & 71 & 73 & 77 & 81 & 83 \\
\hline & 85 & 87 & 91 & 95 & 97 & 99 & 101 & 103 & 105 & 109 & 113 & 115 & 117 & 119 & 123 & 127 \\
\hline & 0 & 4 & 8 & 10 & 12 & 14 & 18 & 22 & 24 & 26 & 28 & 30 & 32 & 36 & 40 & 42 \\
\hline & 44 & 46 & 50 & 54 & 56 & 58 & 60 & 62 & 64 & 68 & 72 & 74 & 76 & 78 & 82 & 86 \\
\hline & 88 & 90 & 92 & 94 & 96 & 100 & 104 & 106 & 108 & 110 & 114 & 118 & 120 & 122 & 124 & 126 \\
\hline $\mathrm{IS}_{2}(+,-,-)$ & 2 & 6 & 11 & 15 & 34 & 38 & 43 & 47 & 66 & 70 & 75 & 79 & 98 & 102 & 107 & 111 \\
\hline $\mathrm{IS}_{2}(-,+,-)$ & 16 & 20 & 25 & 29 & 48 & 52 & 57 & 61 & 80 & 84 & 89 & 93 & 112 & 116 & 121 & 125 \\
\hline
\end{tabular}

\section{a. Experiment 5.1}

By keeping $\lambda=2$ fixed and varying the initial conditions over the 128 vertices of the hypercube labeled from 0 through 127 with $\alpha=0.01$, the model solutions computed using the Runge-Kutta routine in MATLAB are plotted in Figs. 8 and 9. A record of the equilibria to which the solutions converge are given in Table 7. Recall from our earlier discussion in section 3 that each of the steady-state solutions of S-LOM (7) defines a periodic behavior in the physical space.

Figure 8 contains the plot of the solution corresponding to 96 initial conditions that lead to convergence to the equilibria in $\mathrm{IS}_{1}$. Of these, the solutions corresponding to $n_{1}=48$ initial conditions converging to the equilibria in $\mathrm{IS}_{1}(+,-,-)$ are shown in the left column of Fig. 8, and the solutions from the rest of the $n_{2}=48$ initial conditions converging to its image equilibria in $\mathrm{IS}_{1}(-,+,-)$ are shown in the right column of Fig. 8.

Figure 9, likewise, contain the plot of the solutions from the rest of 32 initial conditions converging to the equilibria in $\mathrm{IS}_{2}$. Of these, exactly $m_{1}=16$ solutions converge to $\mathrm{IS}_{2}(+,-,-)$, as shown in the left column of Fig. 9, and the rest $m_{2}=16$ solutions converge to the equilibria in $\operatorname{IS}_{2}(-,+,-)$, as given in the right column of Fig. 9.

Stated in other words, the entries in the Table 7 and the plots in Figs. 8 and 9 together provide a complete picture of the behavior of the ensemble for $\lambda=2$.

\section{b. Experiment 5.2}

By keeping $\alpha=0.01$ as in experiment 5.1 and increasing $\lambda=2.5$, we repeated the analog of experiment 5.1. It turns out in this case, the solution from all the 128 points converge to an equilibrium in $\mathrm{IS}_{2}$ with exactly $n_{1}=64$ converging to $\operatorname{IS}_{2}(+,-,-)$ and the rest converging to $\mathrm{IS}_{2}(-,+,-)$ as shown in Table 8 and Fig. 10.

\section{c. Experiment 5.3}

In this experiment, we explore the behavior of the solution of the S-LOM (7) in (1) and (2) starting from the same initial condition as in Saltzman 1962 paper, namely, $X_{1}(0)=X_{2}(0)=X_{3}(0)=0.0005$ and $X_{4}(0)=$ $X_{5}(0)=X_{6}(0)=X_{7}(0)=0.0$ and when $\lambda=28$. This initial condition is such that there is nonzero initial energy in each of the subspaces $\mathrm{IS}_{1}, \mathrm{IS}_{2}$, and $\mathrm{IS}_{3}$. Since $\lambda=28$, the energy within the subspaces IS $_{1}$ and IS $_{2}$ grow and become chaotic, and the solution in the three subspaces interact through the common dimension $X_{7}$. Phase plots $X_{i}$ versus $X_{j}$ for $i \neq j$ for this case are given in Fig. 11. From the figure, we can easily identify the chaotic behavior in the form of a double attractor, one in each of the subspaces $\mathrm{IS}_{1}$ and $\mathrm{IS}_{2}$ and their interaction with $\mathrm{IS}_{3}$. Variation of the seven Lyapunov exponents $L_{i}, 1 \leq i \leq 7$ for the S-LOM (7) in (1) and (2) with $\lambda$ starting from the same initial condition as in Saltzman (1962) is given in Fig. 12. Positive values of $L_{1}$ and $L_{2}$ further confirm the simultaneous onset of chaos in $\mathrm{IS}_{1}$ and $\mathrm{IS}_{2}$. Also notice that the overall sum of all the seven Lyapunov exponents are negative. It should be

TABLE 8. As in Table 7, but for $\lambda=2.5$.

\begin{tabular}{|c|c|c|c|c|c|c|c|c|c|c|c|c|c|c|c|c|}
\hline \multirow{2}{*}{$\frac{\text { Equilibrium }}{\mathrm{IS}_{2}(+,-,-)}$} & \multicolumn{16}{|c|}{ Encoding of initial conditions } \\
\hline & 2 & 3 & 6 & 7 & 10 & 11 & 14 & 15 & 18 & 19 & 22 & 23 & 26 & 27 & 30 & 31 \\
\hline & 34 & 35 & 38 & 39 & 42 & 43 & 46 & 47 & 50 & 51 & 54 & 55 & 58 & 59 & 62 & 63 \\
\hline & 66 & 67 & 70 & 71 & 74 & 75 & 78 & 79 & 82 & 83 & 86 & 87 & 90 & 91 & 94 & 95 \\
\hline & 98 & 99 & 102 & 103 & 106 & 107 & 110 & 111 & 114 & 115 & 118 & 119 & 122 & 123 & 126 & 127 \\
\hline \multirow{4}{*}{$\operatorname{IS}_{2}(-,+,-)$} & 0 & 1 & 4 & 5 & 8 & 9 & 12 & 13 & 16 & 17 & 20 & 21 & 24 & 25 & 28 & 29 \\
\hline & 32 & 33 & 36 & 37 & 40 & 41 & 44 & 45 & 48 & 49 & 52 & 53 & 56 & 57 & 60 & 61 \\
\hline & 64 & 65 & 68 & 69 & 72 & 73 & 76 & 77 & 80 & 81 & 84 & 85 & 88 & 89 & 92 & 93 \\
\hline & 96 & 97 & 100 & 101 & 104 & 105 & 108 & 109 & 112 & 113 & 116 & 117 & 120 & 121 & 124 & 125 \\
\hline
\end{tabular}



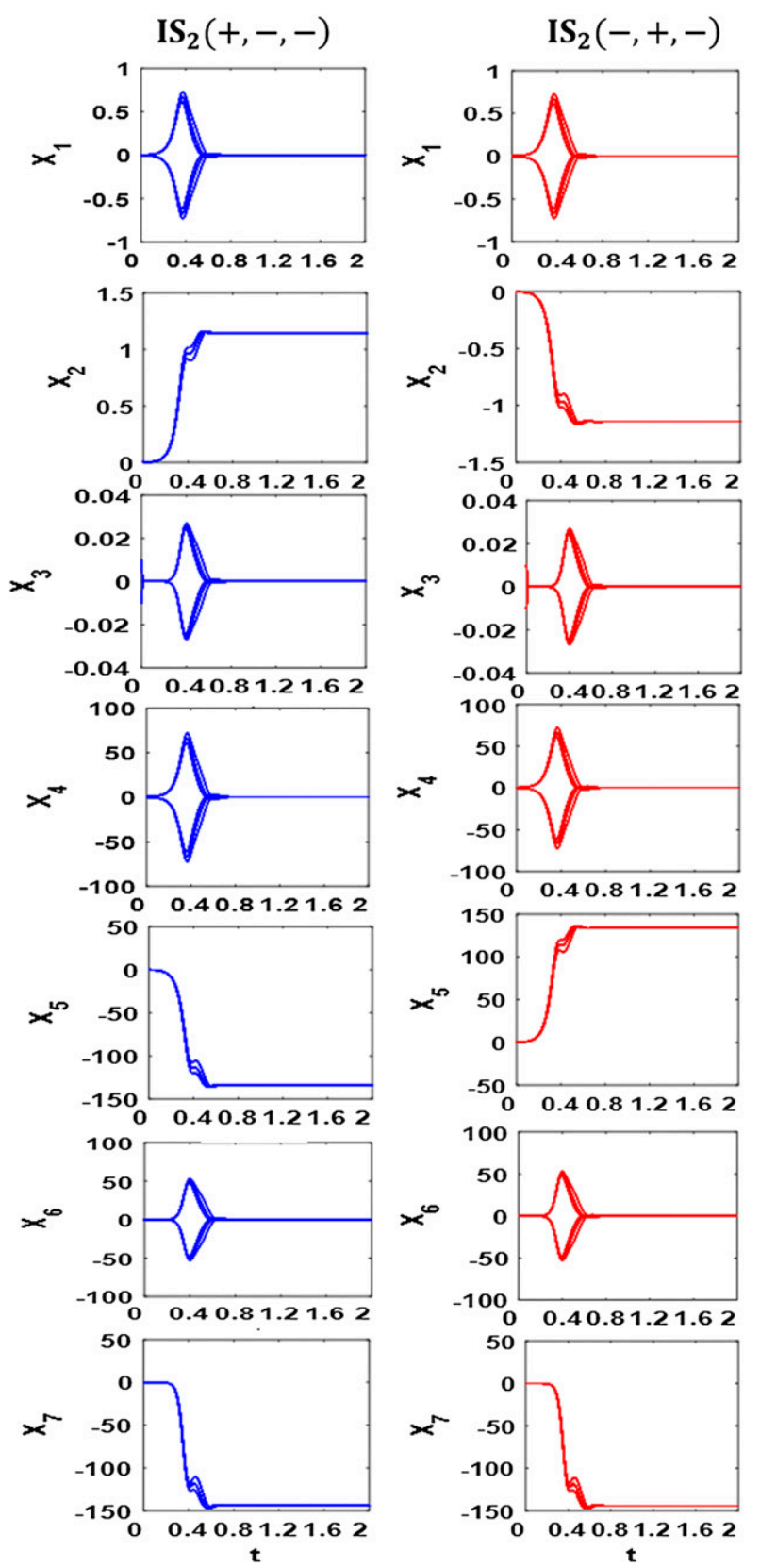

FIG. 10. Plot of the solution of (1) for $\lambda=2.5$ converging to equilibria in $\mathrm{IS}_{2}$.

interesting to identify the different basins of attraction for various values of $\lambda$.

\section{Summary and conclusions}

While our analysis does not add much to the physical aspects of convection that is already contained in Saltzman (1962), it brings completeness to Saltzman's profound contribution to the theory of convective motion in fluids, which is concisely summarized as follows:
1) The state space $R^{7}$ for the S-LOM (7) in (1) is the union of three invariant subspaces $\mathrm{IS}_{1}, \mathrm{IS}_{2}$, and $\mathrm{IS}_{3}$. The presence of the three invariant subspaces is an artifact resulting from the structure of S-LOM (7) in (1) and (2).

2) The phase volume decreases exponentially in time and the solutions to S-LOM (7) remain uniformly bounded for all initial conditions and values of $\lambda$.

3) The S-LOM (7) when projected onto $\mathrm{IS}_{3}$ reduces to an asymptotic stable linear dynamics with the origin as the unique global attractor in $\mathrm{IS}_{3}$.

4) There are two branches of the equilibria-onedimensional manifold parameterized by $\lambda$, in $\mathrm{IS}_{1}$ dented by $\operatorname{IS}_{1}(+,-,-)$ and $\operatorname{IS}_{1}(-,+,-)$, which are reflections of each other with respect to $X_{7}$ axis. Similarly, there are two branches of equilibriaone-dimensional manifold parameterized by $\lambda$, in $\mathrm{IS}_{2}$ dented by $\mathrm{IS}_{2}(+,-,-)$ and $\mathrm{IS}_{2}(-,+,-)$, which are also reflections of each other with respect to $X_{7}$ axis.

5) Chaotic regime: For values of $\lambda>\lambda^{*}$, the solutions in IS $_{1}$ and IS $_{2}$ exhibit chaotic behavior resulting from a Hopf bifurcation. The resulting instability is characterized by the existence of a positive Lyapunov exponent and the fractal dimension of the strange attractor.

6) Periodic solution of Lorenz-like LOM (3) in (4) and (5) and (6) and (7).

Our analysis of the Lorenz-like LOM (3) given by (4) and (5) in $I_{1}$ and that by (6) and (7) in $I_{2}$ thus far concentrated on establishing a set of qualitative similarity measures between these two systems and the Lorenz 1963 model (Lewis et al. 2006, chapter 32) in (10). This list includes (i) the existence of bifurcation of the equilibrium at the origin for values of $\lambda$ close to 1 , (ii) the presence of the $1 \mathrm{D}$ equilibrium manifolds in $\mathrm{IS}_{1}$ and IS $_{2}$ parameterized by $\lambda$, (iii) the existence of Hopf bifurcation for $\lambda=\lambda^{*}$, and (iv) and computation and comparison of Lyapunov exponents and the fractal dimension of the resulting chaotic attractor.

However, a careful review of the classical literature (Sparrow 2012) relating to the analysis of the Lorenz 1963 model reveals that this latter model also exhibits a rich variety of periodic behavior. This observation calls for the analysis of the periodic solutions of the Lorenz-like LOM (3) in (4) and (5) and (6) and (7).

The latter analysis can be done in one of two ways. First is to use a numerical method described in appendix E of Sparrow (2012) for locating a periodic orbit. Clearly, this involves detailed exploration of the state space and the parameter space, 

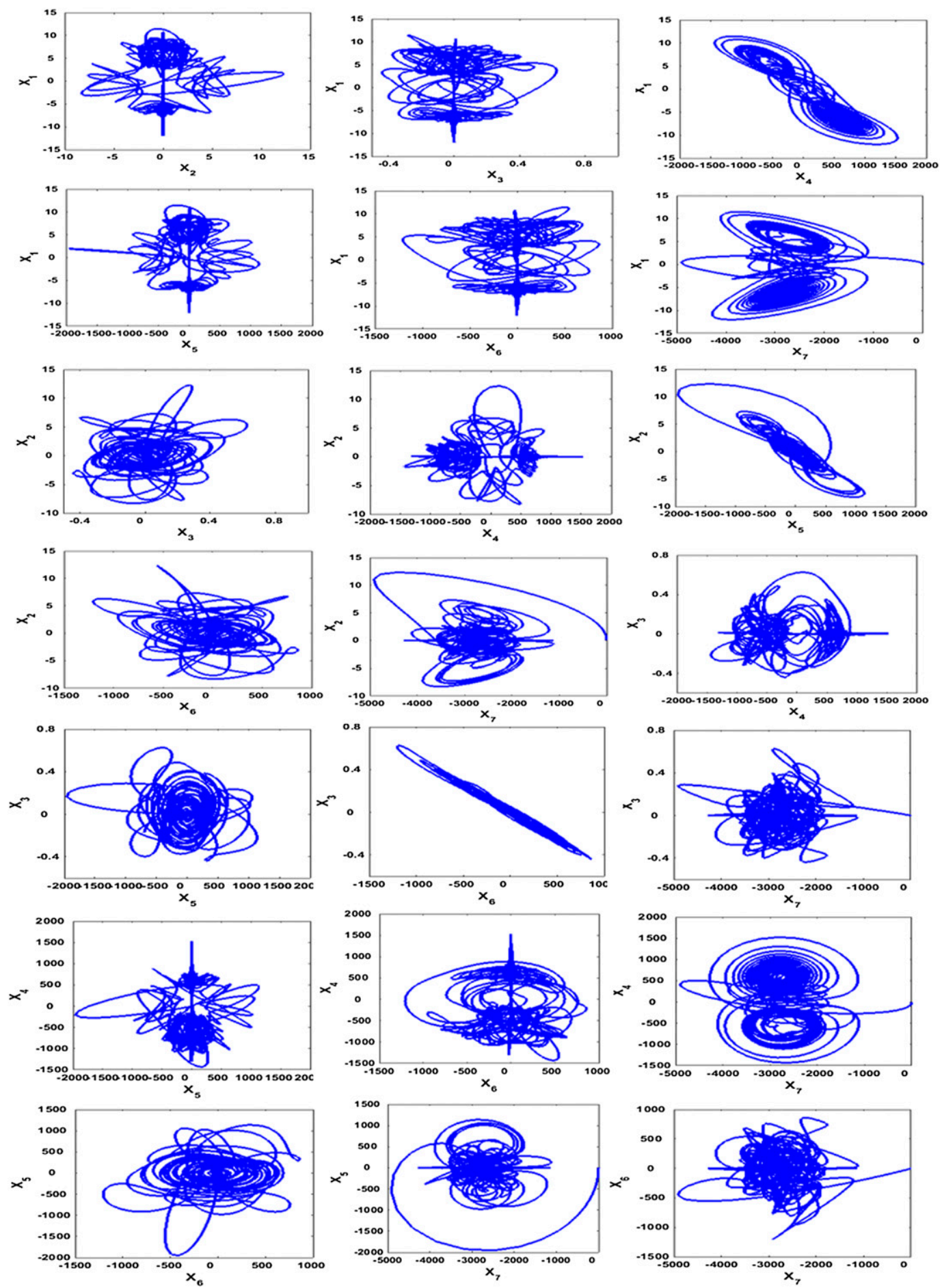

FIG. 11. Phase plot of solution to S-LOM (7) with $X_{1}(0)=X_{2}(0)=X_{3}(0)=0.0005$ and $X_{4}(0)=X_{5}(0)=$ $X_{6}(0)=X_{7}(0)=0.0$ - same initial condition as in Saltzman (1962).

which in principle could be time consuming. A second alternative is to construct an invertible transformation, using which Lorenz-like systems in (4) and (5) and in (6) and (7) can be reduced to the Lorenz system in (10). Indeed, by rescaling the state variables $\left(Y_{1}, Y_{4}, Y_{7}\right)$ and the time variable $t$ in appendix $\mathrm{D}$, we construct a linear invertible transformation that proves the equivalence between LOM 

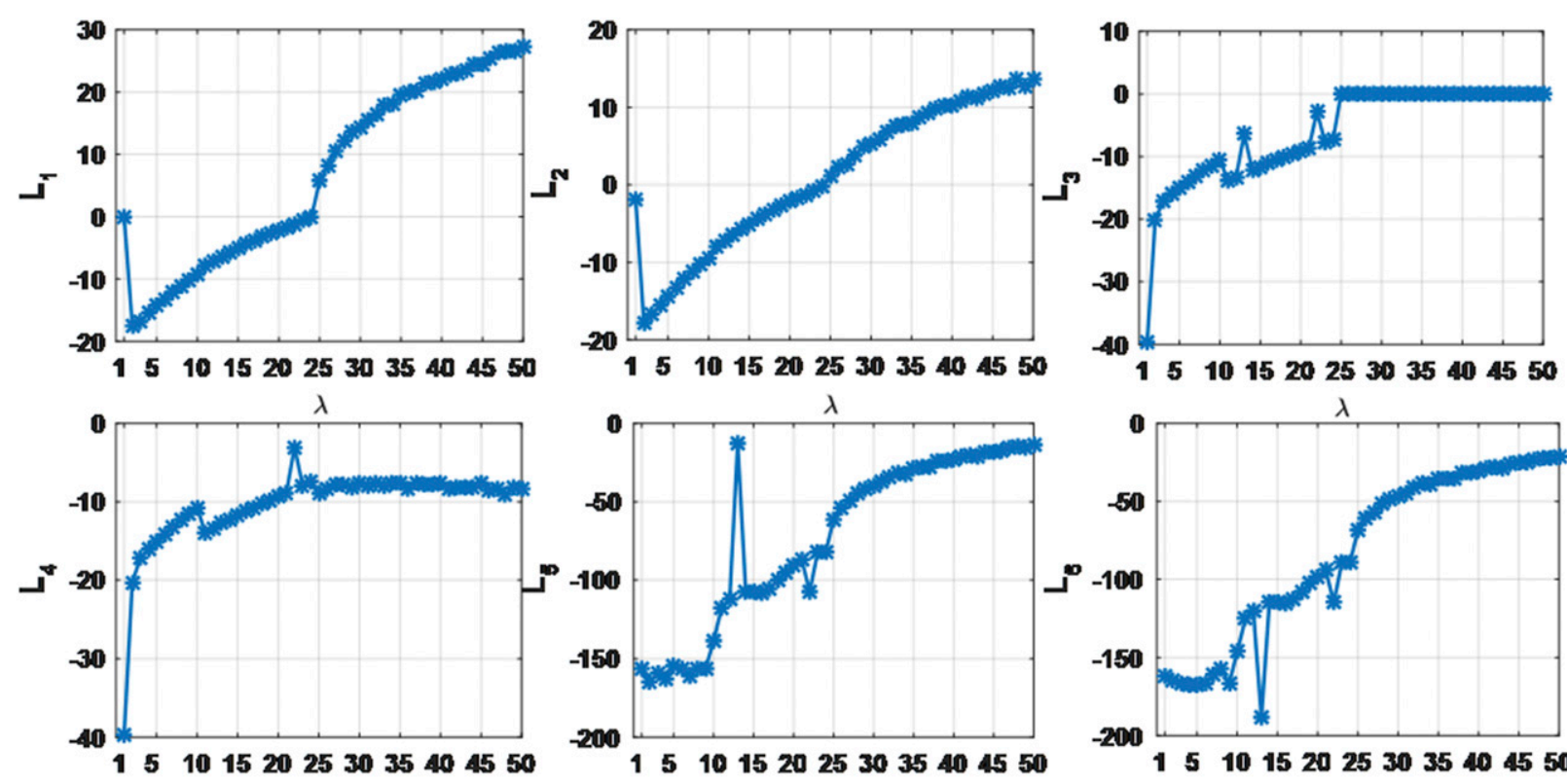

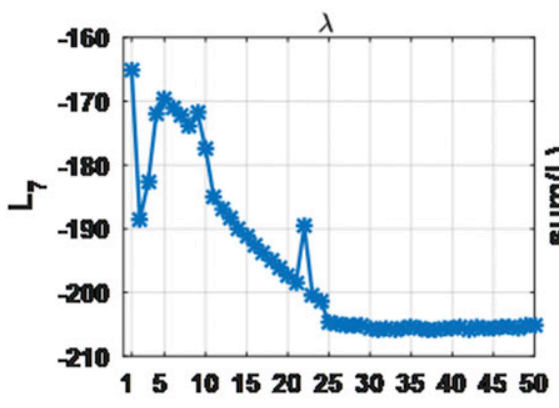

$\lambda$

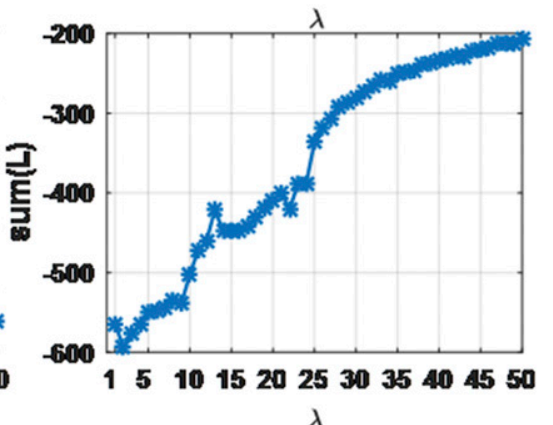

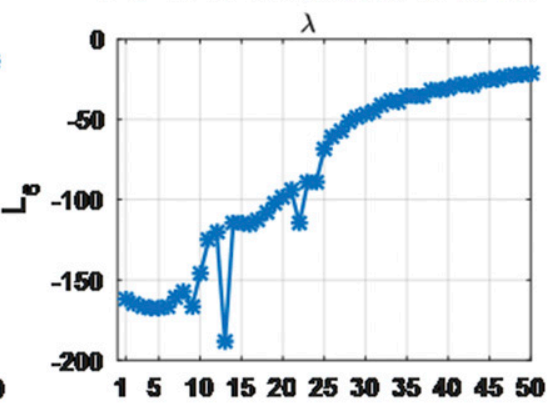

$\lambda$

FIG. 12. Variation of the Lyapunov exponent with $\lambda$ for the S-LOM (7) in (1) and (2) starting from the same initial condition as in Fig. 11, $L_{i}$ refers to the $i$ th exponent and sum $=\sum_{i=1}^{7} L_{i}$.
(3) in (4) and (5) and a specific version of (10) with $\sigma=10, \beta=8 / 3$, where $\rho$ in (10) is related to the $\lambda$ in (4) and (5) by the relation $\rho=0.9993 \lambda$. A similar transformation between (6) and (7) and (10) can be likewise obtained.

7) For general initial conditions with nonzero energy in IS $_{1}$ and IS $_{2}$, the S-LOM (7) in (1) and (2) exhibits simultaneously similar behavior in $\mathrm{IS}_{1}$ and $\mathrm{IS}_{2}$. The presence of the double attractor is noteworthy.

Finally, Saltzman (1962) has had great appeal to readership in fluid mechanics and meteorology. This work has brought some sense of completeness to Saltzman (1962) and should fundamentally be viewed as a tribute to him and a means to expand the usefulness of the family of low-order models for convection.

Acknowledgments. We wish to record our sincere thanks to Qin Xu, National Severe Storms Laboratory, for his questions related to the proof of the boundedness of the solution in appendix A and Alex Gluhosky, Purdue University, for his interest and encouragement.
We are very grateful to the two anonymous reviewers, whose multifaceted questions helped to improve the overall flow and the readability of the paper.

\section{APPENDIX A}

\section{Stability of Equilibrium $E_{1}$ at the Origin}

Analysis of the stability of the equilibrium $E_{1}$ at the origin relates to quantifying the eigenstructure of the sparse, banded Jacobian matrix $\mathbf{J}_{\mathbf{f}}(0)$ in (12), which takes the general form

$$
\mathbf{A}=\left[\begin{array}{ccccccc}
a_{1} & 0 & 0 & b_{1} & 0 & 0 & 0 \\
0 & a_{2} & 0 & 0 & b_{2} & 0 & 0 \\
0 & 0 & a_{3} & 0 & 0 & b_{3} & 0 \\
c_{1} \lambda & 0 & 0 & a_{4} & 0 & 0 & 0 \\
0 & c_{2} \lambda & 0 & 0 & a_{5} & 0 & 0 \\
0 & 0 & c_{3} \lambda & 0 & 0 & a_{6} & 0 \\
0 & 0 & 0 & 0 & 0 & 0 & a_{7}
\end{array}\right]
$$



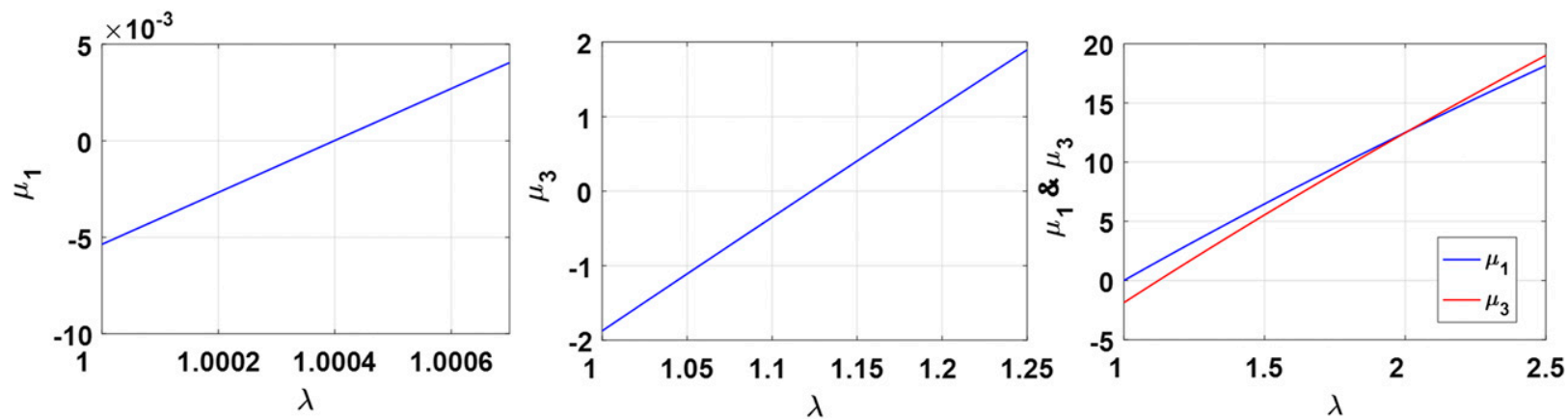

FIG. A1. Plot of the variation of eigenvalues $\mu_{1}$ and $\mu_{3}$ of $\mathbf{A}$ as a function of $\lambda$ : (a) $\mu_{1}$ only, (b) $\mu_{3}$ only, and (c) $\mu_{1}$ and $\mu_{3}$.

where $a_{i}=C_{i i 0}$ for $1 \leq i \leq 7, b_{1}=C_{140}, b_{2}=C_{250}, b_{3}=C_{360}$, $c_{1}=C_{410}, c_{2}=C_{520}$, and $c_{3}=C_{630}$, whose numerical values are given in Table 1 .

Let $\mu \in R$ and $\boldsymbol{\eta} \in R^{7}$ be an eigen pair of $\mathbf{A}$ in (A1), that is, $\mathbf{A} \boldsymbol{\eta}=\mu \boldsymbol{\eta}$. By direct substitution, it can be verified that the sparse, banded structure of $\mathbf{A}$ allows us to naturally decompose the seven-dimensional linear system

$$
\mathbf{A} \boldsymbol{\eta}=\mu \boldsymbol{\eta}
$$

into a collection of four disjoint subsystems each of whose dimension is no more than two, as shown below:

$$
\begin{aligned}
S_{1}:\left(a_{1}-\mu\right) \eta_{1}+b_{1} \eta_{4} & =0, \\
c_{1} \lambda \eta_{1}+\left(a_{4}-\mu\right) \eta_{4} & =0 ; \\
S_{2}:\left(a_{2}-\mu\right) \eta_{2}+b_{2} \eta_{5} & =0, \\
c_{2} \lambda \eta_{2}+\left(a_{5}-\mu\right) \eta_{5} & =0 ; \\
S_{3}:\left(a_{3}-\mu\right) \eta_{3}+b_{3} \eta_{5} & =0, \\
c_{3} \lambda \eta_{3}+\left(a_{6}-\mu\right) \eta_{6} & =0 ;
\end{aligned}
$$

and

$$
S_{4}:\left(a_{7}-\mu\right) \eta_{7}=0
$$

Indeed, by solving these subsystems, we can recover the full eigenstructure $\mathbf{A}$ in (A1).

Solution of $S_{4}$ : Trivially, the seventh eigenvalue $\mu_{7}$ given by $\mu_{7}=a_{7}=C_{770}=-39.479$ is independent of $\lambda$. Its corresponding eigenvector is

$$
\boldsymbol{\eta}=(0,0,0,0,0,0,1)^{\mathrm{T}} \in \mathrm{IS}_{1} \cap \mathrm{IS}_{2} \cap \mathrm{IS}_{3},
$$

which is the seventh standard unit vector in $R^{7}$.

Solution of $S_{1}$ : The two eigenvalues, say, $\mu_{1}$ and $\mu_{2}$ arising from solving the $2 \times 2$ linear system in
(A3) are given by the solution of the characteristic polynomial

$$
p_{1}(\mu)=\mu^{2}-\left(a_{1}+a_{4}\right) \mu+\left(a_{1} a_{4}-\lambda b_{1} c_{1}\right)=0 .
$$

Let $\mu_{1} \geq \mu_{2}$. The variation of $\mu_{1}$ as a function of $\lambda$ is given in Fig. A1a. It can be easily verified that the two (distinct) eigenvectors corresponding to $\mu_{1}$ and $\mu_{2}$ take the general form as

$$
\boldsymbol{\eta}=\left(\eta_{1}, 0,0, \eta_{4}, 0,0,0\right)^{\mathrm{T}} \in \mathrm{IS}_{1},
$$

where $\eta_{1}^{2}+\eta_{4}^{2}=1$. From the definition of the invariant subspace $\mathrm{IS}_{1}$, it is immediate that these two eigenvector are in $\mathrm{IS}_{1}$.

Solution of $S_{2}$ : Following similar reasoning, the next two eigenvalues $\mu_{3}$ and $\mu_{4}$ are obtained by solving the characteristic polynomial

$p_{2}(\mu)=\mu^{2}-\left(a_{2}+a_{5}\right) \mu+\left(a_{2} a_{5}-\lambda b_{2} c_{2}\right)=0$.

Let $\mu_{3} \geq \mu_{4}$. The variation of $\mu_{3}$ as a function of $\lambda$ is given in Fig. A1b. The two (distinct) eigenvectors corresponding to $\mu_{3}$ and $\mu_{4}$ take the general form as

$$
\boldsymbol{\eta}=\left(0, \eta_{2}, 0,0, \eta_{5}, 0,0\right)^{\mathrm{T}} \in \mathrm{IS}_{2},
$$

where $\eta_{2}^{2}+\eta_{5}^{2}=1$. These two eigenvectors belong to the subspace $\mathrm{IS}_{2}$.

Solution of $S_{3}$ : The last two remaining eigenvalues $\mu_{5}$ and $\mu_{6}$ are the roots of the characteristic polynomial

$$
p_{3}(\mu)=\mu^{2}-\left(a_{3}+a_{6}\right) \mu+\left(a_{3} a_{6}-\lambda b_{3} c_{3}\right)=0
$$

of the solution in (A5). It is a simple exercise to verify the eigenvectors for $\mu_{5}$ and $\mu_{6}$ taking the general form 


$$
\boldsymbol{\eta}=\left(0,0, \eta_{3}, 0,0, \eta_{6}, 0\right)^{\mathrm{T}} \in \mathrm{IS}_{3},
$$

where $\eta_{3}^{2}+\eta_{6}^{2}=1$. These two eigenvectors belong to the subspace $\mathrm{IS}_{3}$.

Summary of the stability analysis: From the variation of the seven eigenvalues of $\mathbf{A}$ as a function of $\lambda$, it is immediate that the first bifurcation occurs at $\lambda \approx \lambda_{1}^{*}=1.0004$. Below this value, the origin is a hyperbolic attractor. Above this value, the origin becomes a saddle. More specifically, $\mu_{1}$ becomes positive for $\lambda>1.0004$ and the corresponding unstable eigen direction $\boldsymbol{\eta}=\left(\eta_{1}, 0,0, \eta_{4}, 0,0,0\right)^{\mathrm{T}} \in$ $\mathrm{IS}_{1}$ carries the solution away from the origin. As we increase $\lambda$, around $\lambda \approx \lambda_{2}^{*}=1.1231$, the eigenvalue $\mu_{3}$ of the system $S_{2}$ becomes positive, creating another unstable eigen direction $\boldsymbol{\eta}=$ $\left(0, \eta_{2}, 0,0, \eta_{5}, 0,0\right)^{\mathrm{T}} \in \mathrm{IS}_{2}$. However, from Fig. A1c, it is clear that $\mu_{1}$ remains the dominant eigen value for all $\lambda \leq \lambda_{3}^{*}=2.0050$, but $\mu_{3}$ becomes dominant for $\lambda>\lambda_{3}^{*}$. Hence, for all $\lambda_{1}^{*}<\lambda<\lambda_{2}^{*}$, any solution starting close to the origin moves away from it along the eigenvector $\boldsymbol{\eta}=\left(\eta_{1}, 0,0, \eta_{4}, 0,0,0\right)^{\mathrm{T}}$ in $\mathrm{IS}_{1}$ depending on $\lambda$ and settles down in an equilibrium in $\mathrm{IS}_{1}$. For $\lambda>\lambda_{2}^{*}$, solution close to the origin moves away from it either along $\left(\eta_{1}, 0,0, \eta_{4}, 0,0,0\right)^{\mathrm{T}} \in \mathrm{IS}_{1}$ or along $\left(0, \eta_{2}, 0,0, \eta_{5}, 0,0\right)^{\mathrm{T}} \in \mathrm{IS}_{2}$, depending on both the initial condition and value of $\lambda$.

For $\lambda>28$, the system becomes chaotic but remains within $\mathrm{IS}_{1}$ or $\mathrm{IS}_{2}$, depending on the initial condition.

\section{APPENDIX B}

\section{Boundedness of the Solution of S-LOM (7)}

Consider a quadratic energy function $E: R^{7} \rightarrow R$ given by

$$
E(\mathbf{X})=\frac{1}{2} \mathbf{X}^{\mathrm{T}} \mathbf{K} \mathbf{X}
$$

where $\mathbf{K} \in R^{7 \times 7}$ is a diagonal matrix:

$$
\mathbf{K}=\operatorname{Diag}\left(K_{1}, K_{2}, \ldots, K_{7}\right)
$$

with positive diagonal entries. Since the S-LOM (7) in (2) is a forced, dissipative system, $E(\mathbf{X})$ cannot be a constant along its trajectory.

Our goal is to choose the diagonal elements of $\mathbf{K}$ such that the sign of $\dot{E}(\mathbf{X})$ can be conveniently and analytically evaluated. Computing the time derivative of $E(\mathbf{X})$ along the trajectory of (2) and collecting the like terms, we obtain

$$
\begin{aligned}
\dot{E}(\mathbf{X})= & \sum_{i=1}^{7} \mathbf{K} X_{i} \dot{X}_{i} \\
= & X_{1} X_{2} X_{3}\left[K_{1} C_{123}+K_{2} C_{213}+K_{3} C_{312}\right] \\
& +X_{3} X_{4} X_{5}\left[K_{4} C_{435}+K_{5} C_{534}\right] \\
& +X_{2} X_{4} X_{6}\left[K_{4} C_{426}+K_{6} C_{624}\right] \\
& +X_{2} X_{5} X_{7}\left[K_{5} C_{527}+K_{7} C_{725}\right] \\
& +X_{1} X_{4} X_{7}\left[K_{4} C_{147}+K_{7} C_{714}\right] \\
& +X_{1} X_{5} X_{6}\left[K_{5} C_{516}+K_{6} C_{615}\right] \\
& +X_{1} X_{4}\left[K_{1} C_{140}+K_{4} C_{410} \lambda\right] \\
& +X_{2} X_{5}\left[K_{2} C_{250}+K_{5} C_{520} \lambda\right] \\
& +X_{3} X_{6}\left[K_{3} C_{360}+K_{6} C_{630} \lambda\right] \\
& +K_{1} C_{110} X_{1}^{2}+K_{2} C_{220} X_{2}^{2}+K_{3} C_{330} X_{3}^{2} \\
& +K_{4} C_{440} X_{4}^{2}+K_{5} C_{550} X_{5}^{2}+K_{6} C_{660} X_{6}^{2} \\
& +K_{7} C_{770} X_{7}^{2},
\end{aligned}
$$

which is the sum of 6 cubic and 10 quadratic terms.

Since

$$
\begin{array}{ll}
C_{435}=-C_{534}, & 2 C_{527}=-C_{725}, \\
C_{426}=-C_{624}, & 2 C_{417}=-C_{714}, \\
C_{516}=-C_{615}, &
\end{array}
$$

setting

$$
K_{4}=K_{5}=K_{6}=2 K_{7}=1,
$$

the coefficients of the five cubic terms except that of $X_{1}, X_{2}$, and $X_{3}$ vanish. Now setting

$$
K_{1} C_{123}+K_{3} C_{312}=K_{2}\left|C_{213}\right|
$$

or

$$
\left(\frac{K_{1}}{K_{2}}\right) \frac{C_{123}}{\left|C_{213}\right|}+\left(\frac{K_{3}}{K_{2}}\right) \frac{C_{312}}{\left|C_{213}\right|}=1
$$

that is,

$$
\frac{\left(K_{1} / K_{2}\right)}{0.9366}+\frac{\left(K_{3} / K_{2}\right)}{14.1123}=1,
$$

we force the coefficient of the remaining cubic terms $X_{1}, X_{2}$, and $X_{3}$ also to zero. Clearly, there are infinitely many choices for $K_{1}, K_{2}$, and $K_{3}$ satisfying (B7).

Assuming that the values of $K_{i}$ are chosen to satisfy (B5) and (B7), we now regroup the remaining 10 quadratic terms into three groups consistent with the 
properties of the invariant subspaces $\mathrm{IS}_{1}, \mathrm{IS}_{2}$, and $\mathrm{IS}_{3}$ in section 2. Accordingly,

$$
\begin{aligned}
\dot{E}(\mathbf{X})= & Q_{14}\left(X_{1}, X_{4}\right)+Q_{25}\left(X_{2}, X_{5}\right)+Q_{36}\left(X_{3}, X_{6}\right) \\
& +Q_{7}\left(X_{7}\right),
\end{aligned}
$$

where

$$
\begin{aligned}
Q_{14}\left(X_{1}, X_{4}\right)= & K_{1} C_{110} X_{1}^{2}+K_{4} C_{440} X_{4}^{2}+X_{1} X_{4}\left[K_{1} C_{140}\right. \\
& \left.+K_{4} C_{410} \lambda\right], \\
Q_{25}\left(X_{2}, X_{5}\right)= & K_{2} C_{220} X_{2}^{2}+K_{5} C_{550} X_{5}^{2}+X_{2} X_{5}\left[K_{2} C_{250}\right. \\
& \left.+K_{5} C_{520} \lambda\right], \\
Q_{36}\left(X_{3}, X_{6}\right)= & K_{3} C_{330} X_{3}^{2}+K_{6} C_{660} X_{6}^{2}+X_{3} X_{6}\left[K_{3} C_{360}\right. \\
& \left.+K_{6} C_{630} \lambda\right],
\end{aligned}
$$

and

$$
Q_{7}\left(X_{7}\right)=K_{7} C_{770} X_{7}^{2} .
$$

From Table 1 , since $C_{770}=-39.479, Q_{7}$ in (B9) is negative definite. To analyze the negative definiteness of the rest of the three quadratic forms in (B9), we invoke two basic facts from the theory of quadratic forms.

Fact 1: If $\mathbf{A} \in R^{n \times n}$, then $\mathbf{A}=\mathbf{A}_{s}+\mathbf{A}_{s s}$, where the symmetric part $\mathbf{A}_{s}$ and the skew-symmetric part $\mathbf{A}_{s s}$ are given by $\mathbf{A}_{s}=(1 / 2)\left(\mathbf{A}+\mathbf{A}^{\mathrm{T}}\right)$ and $\mathbf{A}_{s s}=$ $(1 / 2)\left(\mathbf{A}-\mathbf{A}^{\mathrm{T}}\right)$

Fact 2: From $\mathbf{X}^{\mathrm{T}} \mathbf{A} \mathbf{X}=\mathbf{X}^{\mathrm{T}} \mathbf{A}^{\mathrm{T}} \mathbf{X}$, it is immediate that $\mathbf{X}^{\mathrm{T}} \mathbf{A} \mathbf{X}=\mathbf{X}^{\mathrm{T}} \mathbf{A}_{s} \mathbf{X}$.

Sign definiteness of $Q_{14}$ : Using these facts,

$$
Q_{14}=\left(\begin{array}{ll}
X_{1} & X_{4}
\end{array}\right)\left[\begin{array}{ll}
a_{1} & b_{1} \\
b_{1} & c_{1}
\end{array}\right]\left(\begin{array}{l}
X_{1} \\
X_{4}
\end{array}\right)
$$

with $a_{1}=K_{1} C_{110}, c_{1}=K_{4} C_{440}$, and $2 b_{1}=K_{1} C_{140}+$ $K_{4} C_{410} \lambda$. Hence, $Q_{14}$ is negative definite when

$$
a_{1} c_{1}<b_{1}^{2} .
$$

Substituting for $a_{1}, b_{1}, c_{1}$ and using the values of $C_{i j k}$ from Table 1 , since $K_{4}=1$, (B11) reduces to

$$
4 K_{1} \leq\left(0.032 K_{1}+31.198 \lambda\right)^{2} .
$$

Sign definiteness of $Q_{25}$ : Again, from

$$
Q_{25}=\left(\begin{array}{ll}
X_{2} & X_{5}
\end{array}\right)\left[\begin{array}{ll}
a_{2} & b_{2} \\
b_{2} & c_{2}
\end{array}\right]\left(\begin{array}{l}
X_{2} \\
X_{5}
\end{array}\right),
$$

with $a_{2}=K_{2} C_{220}, c_{2}=K_{5} C_{550}$, and $2 b_{2}=K_{2} C_{250}+$ $K_{5} C_{520} \lambda$, it follows that $Q_{25}$ is negative definite if $a_{2} c_{2}<b_{2}^{2}$. Substituting for $a_{2}, b_{2}, c_{2}$ and using the values of $C_{i j k}$ from Table 1 , since $K_{5}=1, Q_{25}$ is negative definite when

$$
4 K_{2} \leq\left(0.02696 K_{2}+33.04 \lambda\right)^{2} .
$$

Sign definiteness of $Q_{36}$ : By similar arguments

$$
Q_{36}=\left(\begin{array}{ll}
X_{3} & X_{6}
\end{array}\right)\left[\begin{array}{ll}
a_{3} & b_{3} \\
b_{3} & c_{3}
\end{array}\right]\left(\begin{array}{l}
X_{3} \\
X_{6}
\end{array}\right),
$$

with $a_{3}=K_{3} C_{330}, c_{3}=K_{6} C_{660}$, and $2 b_{3}=K_{3} C_{360}+$ $K_{6} C_{630} \lambda, Q_{36}$ is negative definite if $a_{3} c_{6}<b_{3}^{2}$. Substituting for $a_{3}, b_{3}, c_{3}$ and using the values of $C_{i j k}$ from Table 1 , since $K_{6}=1, Q_{36}$ is negative definite when

$$
4 K_{3} \leq\left(0.0014 K_{3}+3.667 \lambda\right)^{2} .
$$

Stated in other words, all the four quadratic forms in (B9) are simultaneously negative definite if there exist constants $K_{1}, K_{2}$, and $K_{3}$ that satisfy (B7), (B12), (B14), and (B16) simultaneously.

Setting $K_{2}=1 / 6$ in (B7), it follows that $0 \leq K_{1} \leq$ 0.1561 and $0 \leq K_{3} \leq 2.3521$. Using the standard majorization $^{\mathrm{B} 1}$ argument, inequalities in (B12), (B14), and (B16) hold if

$$
\begin{aligned}
4 \times 0.1561 & =0.6244 \leq(31.198 \lambda)^{2}, \\
4 \times \frac{1}{6} & =0.6667 \leq(33.04 \lambda)^{2}, \\
4 \times 2.3521 & =9.408 \leq(3.667 \lambda)^{2} .
\end{aligned}
$$

Clearly, (B17) holds for all $\lambda \geq 1$. Hence, $\dot{E}(\mathbf{X}) \leq 0$ and the solution of S-LOM (7) in (2) is bounded for all $\lambda \geq 1$.

\section{APPENDIX C}

\section{An Encoding of the Ensemble Members}

In this appendix, we develop a succinct encoding scheme to represent the set of all 128 initial conditions, which correspond to the corners of the sevendimensional hypercube that is centered at the origin with the length of the sides equal to $2 \alpha$ for some $\alpha \in(0,1]$. An example of such a hypercube in $2 \mathrm{D}$ is given in Fig. C1.

The seven-dimensional hypercube of interest has $2^{7}=$ 128 corners. The coordinates of each of these corners corresponds to a string of \pm 1 of length 7 . The encoding

\footnotetext{
${ }^{\mathrm{B} 1}$ The maximum of the lhs is less than the minimum of the rhs.
} 


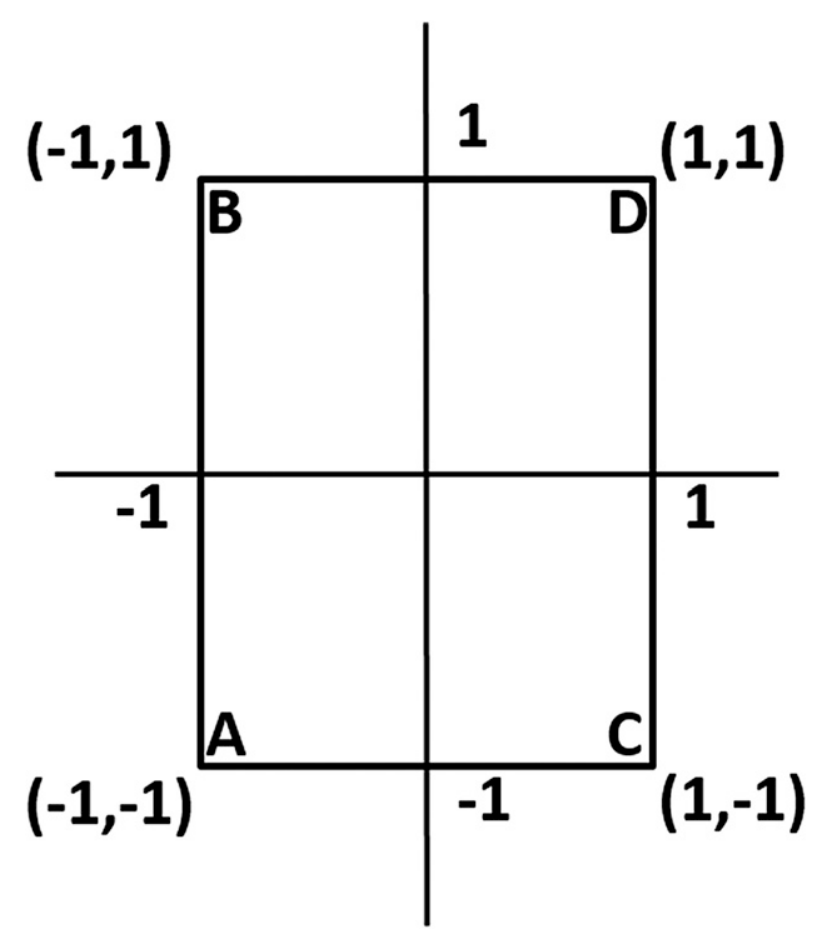

FIG. C1. A 2D hypercube of side length $=2$ centered at the origin .

scheme that enumerates these 128 corners is given below:

1) Let $i$ be an integer in the range 0 to 127 .

2) Let $i=b_{7} b_{6} b_{5} b_{4} b_{3} b_{2} b_{1}$ be the binary representation of the integer $i$.

3) Define an encoding function $\eta$ as follows:

$$
\begin{aligned}
& \eta(1)=1 \\
& \eta(0)=-1
\end{aligned}
$$

4) Then associate the label $i$ with the new string of \pm 1 obtained as follows:

$$
\begin{aligned}
i \rightarrow \eta(i) & =\eta\left(b_{7}\right) \eta\left(b_{6}\right) \eta\left(b_{5}\right) \eta\left(b_{4}\right) \eta\left(b_{3}\right) \eta\left(b_{2}\right) \eta\left(b_{1}\right) \\
& =c_{7} c_{6} c_{5} c_{4} c_{3} c_{2} c_{1},
\end{aligned}
$$

where $c_{j}=\eta\left(b_{j}\right)= \pm 1$, depending on $b_{j}=1$ or 0 .

Accordingly, the four corners of the 2D hypercube in Fig. $\mathrm{C} 1$ is given in Table $\mathrm{C} 1$. That is, the four nodes $\mathrm{A}$, $\mathrm{B}, \mathrm{C}$, and D are encoded as $0,1,2$, and 3 in Table C1. We can likewise develop a table for the 128 nodes of the seven-dimensional hypercube of interest. As an example, consider $i=83$. In binary, $i=83$ is 1010011 . Since $\eta(i)=(1,-1,1,-1,-1,1,1)$, the corner of the hypercube with these coordinates is denoted by the integer $i=83$.
TABLE C1. The four corners of the 2D hypercube in Fig. C1.

\begin{tabular}{rrrrrc}
\hline \hline$i$ & $b_{2} b_{1}$ & \multicolumn{2}{c}{$c_{2} c_{1}$} & Label \\
\hline 0 & 0 & 0 & -1 & -1 & $\mathrm{~A}$ \\
1 & 0 & 1 & -1 & 1 & $\mathrm{~B}$ \\
2 & 1 & 0 & 1 & -1 & $\mathrm{C}$ \\
3 & 1 & 1 & 1 & 1 & $\mathrm{D}$ \\
\hline
\end{tabular}

\section{APPENDIX D}

\section{Equivalence between the Projected S-LOM (7) onto IS 1 Given by (4) and (5) and the Lorenz 1963 Model Given in (10)}

Define a set of new Fourier amplitude variables

$$
\eta_{i}=\frac{Y_{i}}{\alpha_{i}}, \quad i=1,4,7
$$

and a new time variable

$$
\tau=\frac{t}{t_{c}}
$$

where $\alpha_{1}, \alpha_{4}$, and $\alpha_{7}$ are the Fourier amplitude-scale factors and $t_{c}$ is the time-scale factor.

Using (D1) and (D2) in (4) and (5), after simplification, we get the transformed version of the reduced dynamics in $\mathrm{IS}_{1}$ as

$$
\frac{d Y_{i}}{d t}=\frac{d\left(\alpha_{i} \eta_{i}\right)}{d \tau} \frac{d \tau}{d t}=\left(\frac{\alpha_{i}}{t_{c}}\right) \frac{d \eta_{i}}{d \tau}
$$

and

$$
\frac{d\left(\eta_{i}\right)}{d \tau}=\bar{F}_{i}(\boldsymbol{\eta}, \lambda),
$$

where

$$
\begin{aligned}
\bar{F}_{i}(\boldsymbol{\eta}, \lambda)= & \left(\frac{C_{140} t_{c} \alpha_{4}}{\alpha_{1}}\right) \eta_{4}+\left(C_{110} t_{c}\right) \eta_{1} \\
\overline{F_{4}}(\boldsymbol{\eta}, \lambda)= & \left(\frac{C_{417} t_{c} \alpha_{1} \alpha_{7}}{\alpha_{4}}\right) \eta_{1} \eta_{7}+\left(\frac{C_{410} t_{c} \lambda \alpha_{1}}{\alpha_{4}}\right) \eta_{1} \\
& +\left(C_{440} t_{c}\right) \eta_{4} \\
\overline{F_{7}}(\boldsymbol{\eta}, \lambda)= & \left(\frac{C_{714} t_{c} \alpha_{1} \alpha_{4}}{\alpha_{7}}\right) \eta_{1} \eta_{4}+\left(C_{770} t_{c}\right) \eta_{7} .
\end{aligned}
$$

Recall that the values of the coefficients $C_{i j k}$ extracted from Saltzman (1962) are given in Table 1. Now setting $\left(\eta_{1}, \eta_{4}, \eta_{7}\right)=(x, y, z)$, our goal is to choose the four scaling factors $\alpha_{1}, \alpha_{4}, \alpha_{7}$, and $t_{c}$ such that the vector field of (D4) and (D5) matches that of Lorenz model given in (10).

Equating the coefficients of the like terms on the right-hand side of (D5) with that of the Lorenz model 
in (10), we obtain the following set of seven equations relating the above four scaling parameters and the three parameters $\sigma, \beta$, and $\rho$ in the Lorenz model:

$$
\begin{aligned}
t_{c} & =-\frac{\sigma}{C_{110}}, \quad \frac{\alpha_{4}}{\alpha_{1}}=-\frac{C_{110}}{C_{140}}, \\
\alpha_{7} & =-\frac{C_{110}^{2}}{C_{417} C_{140}}, \quad \rho=\frac{C_{410} C_{140} \sigma \lambda}{C_{110}^{2}}, \\
\sigma & =\frac{C_{110}}{C_{440}}, \quad \alpha_{1} \alpha_{4}=\frac{C_{110}^{2}}{C_{714} C_{417} C_{140} \sigma}, \quad \text { and } \\
\beta & =\frac{C_{770} \sigma}{C_{110}} .
\end{aligned}
$$

Substituting the value of $C_{i j k}$ from Table 1 in (D6), we readily obtain the following:

$$
\begin{aligned}
\sigma & =9.999 \approx 10, \quad \beta=2.666 \approx 8 / 3, \quad \rho=0.9993 \lambda, \\
\alpha_{1} & =0.1949, \quad \alpha_{4}=19.2406, \quad \alpha_{7}=1046.837, \quad \text { and } \\
t_{c} & =0.0675 .
\end{aligned}
$$

Stated in other words, there exists an invertible linear scaling of the Fourier amplitudes and the time given in (D1) and (D2), using which we can transform the reduced S-LOM (3) given in (4) and (5) [obtained by projecting S-LOM (7) onto the invariant subspace IS $_{1}$ ] to the Lorenz 1963 model with a specific value of the Prandtl number $\sigma=10$, aspect ratio $\beta=8 / 3$, and the Rayleigh parameters related by $\rho=0.9993 \lambda$.

By using a similar line of argument, we can show that the S-LOM (3) in (6) and (7) is indeed equivalent to the Lorenz model in (10).

\section{REFERENCES}

Arnold, V. I., 1992: Ordinary Differential Equations. Springer, $334 \mathrm{pp}$.

Canuto, C., A. Quarteroni, M. Y. Hussaini, and T. A. Zang, 2007: Spectral Methods: Fundamentals in Single Domain. Springer, $581 \mathrm{pp}$.
Chandrasekhar, S., 1961: Hydrodynamic and Hydromagnetic Stability. Clarendon Press, 654 pp.

Curry, J. H., 1978: A generalized Lorenz system. Commun. Math. Phys., 60, 193-204, https://doi.org/10.1007/BF01612888.

Gilmore, R., and C. Letellier, 2007: The Symmetry of Chaos. Oxford University Press, $545 \mathrm{pp}$.

Gluhovsky, A., and C. Tong, 1999: The structure of energy conserving low-order models. Phys. Fluids, 11, 334-343, https:// doi.org/10.1063/1.869883.

Grassberger, P., and I. Procaccia, 1983: Measuring the strangeness of strange attractors. Physica D, 9, 189-208, https://doi.org/ 10.1016/0167-2789(83)90298-1.

Hirsch, M. W., and S. Smale, 1973: Differential Equations, Dynamical Systems and Linear Algebra. Academic Press, 358 pp.

Lakshmivarahan, S., and Y. Wang, 2008: On the relation between energy-conserving low-order models and a system of coupled generalized Volterra gyrostats with nonlinear feedback. J. Nonlinear Sci., 18, 75-97, https://doi.org/10.1007/ s00332-007-9006-6.

Lewis, J. M., S. Lakshmivarahan, and S. Dhall, 2006: Dynamic Data Assimilation: A Least Squares Approach. Vol. 13. Cambridge University Press, $654 \mathrm{pp}$.

Lorenz, E. N., 1963: Deterministic nonperiodic flow. J. Atmos. Sci. 20, 130-141, https://doi.org/10.1175/1520-0469(1963)020<0130: $\mathrm{DNF}>2.0 . \mathrm{CO} ; 2$.

, 1993: The Essence of Chaos. University Of London, 227 pp.

Malkus, W. V. R., and G. Veronis, 1958: Finite amplitude cellular convection. J. Fluid Mech., 4, 225-260, https://doi.org/10.1017/ S0022112058000410.

Rayleigh, L., 1916: On convection currents in a horizontal layer of fluid, when higher temperature is on the under side. London Edinburgh Dublin Philos. Mag. J. Sci. 32, 529-546, https:// doi.org/10.1080/14786441608635602.

Saltzman, B., 1962: Finite amplitude free convection as an initial value problem-I. J. Atmos. Sci., 19, 329-341, https://doi.org/ 10.1175/1520-0469(1962)019<0329:FAFCAA > 2.0.CO;2.

Shen, J., T. Tang, and L. L. Wang, 2011: Spectral Methods: Algorithms, Analysis and Applications. Springer, 472 pp.

Sparrow, C., 2012: The Lorenz Equations: Bifurcations, Chaos, and Strange Attractors. Vol. 41. Springer, 270 pp.

Tong, C., 2009: Lord Kelvin's gyrostat and its analogs in physics, including the Lorenz model. Amer. J. Phys., 77, 526-537, https://doi.org/10.1119/1.3095813.

Turner, J. S., 1973: Buoyancy Effects in Fluids. Cambridge University Press, $367 \mathrm{pp}$.

Wolf, A., J. B. Swift, H. L. Swinney, and J. A. Vastano, 1985: Determining Lyapunov exponents from a time series. Physica D, 16, 285-317, https://doi.org/10.1016/0167-2789(85)90011-9. 